\title{
LE PRINCIPE DE HUYGHENS DANS LE CAS DE QUATRE VARIABLES INDÉPENDANTES.
}

\author{
PAR
}

\section{J. HADAMARD}

à PARIS.

Dans un précédent travail ${ }^{1}$, j'ai développé certaines conséquences du principe de Huyghens dans le cas d'une équation aux dérivées partielles du second ordre à un nombre impair quelconque de variables indépendantes.

Il s'agit, en l'espèce, de la première forme de ce principe, celle que j'avais désignée précédemment par la lettre $A^{2}$ et qu'on peut appeler la "majeure" de Huyghens (le principe de Huyghens, dans son ensemble, étant considéré comme un syllogisme). On considère un phénomène naturel se déroulant dans l'espacetemps à un nombre quelconque $m$ de dimensions et caractérisé par une fonction unique $u$ des coordonnées, laquelle est supposée régie par une équation aux dérivées partielles linéaire du second ordre

$$
F(u)=\sum_{i, k} A_{i k} \frac{\partial u}{\partial x_{i} \partial x_{k}}+\sum B_{i} \frac{\partial u}{\partial x_{i}}+C u=\varphi
$$

(où les $A_{i k}$, les $B_{i}, C$ et $\varphi$ sont des fonctions données des variables indépendantes $\left.x_{1}, x_{2}, \ldots, x_{m}\right)$ compatible avec la propagation par ondes, c'est à dire appartenant au type hyperbolique normal. ${ }^{3}$

1 "Principe de Huyghens et prolongement analytique", Bull. Soc. Math. France, tome LII, I924, page I4I-178. Ce travail sera, dans la suite, désigné par la lettre $P$.

${ }^{2}$ Conférence à l'occasion du Cinquantenaire de la Société Mathématique de France en I924. Bull. Soc. Math. Fr., t. LII, p. 6ro-640. Ce travail sera désigné par la lettre $C$.

* Voir notre précédent Mémoire des Acta, tome XXXI, 1908 page 333 à 380 (travail que nous désignerons par $A M$ ) particulièrement no 15, page 352, et nos Lectures on Cauchy's problem in partial differential equations, New Haven-London, 1923 (travail qui sera désigné par $Y$ ), 
Soient, d'autre part, $S_{1}, S_{\mathbf{z}}$ deux hypersurfaces dûment orientées $\left(Y, \mathbf{n}^{\mathbf{0}} \mathbf{2 7}\right)$ par rapport au cônes caractéristiques de l'équation; et soit $\left(a_{1}, a_{2}, \ldots a_{m}\right)$ un point de l'hyperespace à $m$ dimensions, point que, comme dans le travail précédemment cité $(P)$, nous nommerons le point o et où l'on se propose de calculer la valeur de $u$. Nous supposerons, comme dans nos recherches précédentes, que le conoïde caractéristique ayant pour sommet ce point découpe, tant sur $S_{1}$ que sur $S_{z}$, des portions limitées en tous sens et, d'une manière générale, que les relations géométriques qualitatives entre les diverses parties des figures que nous aurons à tracer seront les mêmes que si l'on avait à faire à l'équation des ondes sphériques, les hypersurfaces $S_{1}, S_{2}$ n'étant autres que des hyperplans $t=$ const.

Supposons encore, quoique ce ne soit pas strictement nécessaire, que les multiplicités $S_{1}, S_{2}$, ou plutot leurs portions situées à l'intérieur du conoïde caractéristique et auxquelles il est expressément entendu que nous les réduisons désormais, ne se coupent pas, $S_{1}$ étant située entre $S_{2}$ et le sommet o du conoïde.

La solution $u$ de l'équation sera définie si l'on se donne, le long de $S_{z}$, les données de Cauchy, c'est à dire, en un point quelconque 2 de cette multiplicité, la valeur $u_{2}$ de l'inconnue et la valeur $u_{2}^{\prime}$ de sa "dérivée transversale". ${ }^{\prime}$

Sa valeur au point o, par exemple, sera donnée par les formules $(A M$, formules (38), (62); $Y$, formule (39), $\mathbf{n}^{\circ} \mathbf{1 0 5} ;(28)-(30), \mathrm{n}^{\text {os }} \mathbf{1 4 5}-\mathbf{1 4 7}$ ) qui résultent de notre théorie précédente et qui sont de forme différente suivant que le nombre des variables indépendantes est pair ou impair. Dans ces formules, nous rappelons que l'on désigne:

$\operatorname{par} \boldsymbol{A}$, la forme caractéristique $\Sigma A_{i k} \pi_{i} \pi_{k}$ relative à l'équation;

par $\boldsymbol{H}=\Sigma H_{i k} x_{i}^{\prime} x_{k}^{\prime}$, la forme réciproque de la précédente (forme adjointe de $\boldsymbol{A}$ divisée par le discriminant de $\boldsymbol{A}$ );

par $I$, le carré de la distance géodésique de deux points, comptée relativement à l'élément linéaire $H\left(d x_{1}, d x_{2}, \ldots d x_{m} ; x_{1}, x_{2}, \ldots x_{m}\right)$. Par exemple, $\Gamma_{02}$ désignera le carré de la distance géodésique entre les point o et 2 . Egalée à zéro, cette quantité fournit comme lieu de l'un des deux points, lorsque l'autre est donné, le conö̈de caractéristique ayant pour sommet ce point. A l'intérieur du dit conoïde, la quantité $I$ est positive;

par $v$, la solution élémentaire formée à l'aide des deux points, supposés en

no 22, page 39. Dans $A M$, le lecteur est prié d'effectuer les corrections mentionnées au tome 45, fascicule $\mathrm{I}-2$ des Acta. D'antre part, la notation de $Y$ et celle du présent travail diffèrent de celle de $A M$ par le changement de $\mathcal{V}$ en $-\mathcal{V}$.

$1 Y, 40$; dérivée conormale de M. d'Adbémar, ef. $A M$, p. 335. 
Le principe de Huyghens dans le cas de quatre variables indépendantes. 205 situation telle que la quantité $\Gamma$ correspondante soit positive. Lorsque le nombre des variables indépendantes est impair, la quantité $v$ s'exprime par une fraction ayant pour dénominateur un radical (à savoir une puissance impaire de $\sqrt{\Gamma}$ ). $\mathrm{Si}$, au contraire, ce nombre $m$ est pair, elle est de la forme

$$
\frac{V}{\Gamma^{\frac{m}{2}-1}}-\mathcal{V} \log \Gamma
$$

les fonctions $V$ et $\mathcal{V}$ étant définies et régulières (elles sont holomorphes si l'équation est à coefficients analytiques et holomorphes), tout au moins dès que les deux points dont doit dépendre $v$ sont suffisamment voisins l'un de l'autre. Ces quantités existent done quel que soit le signe de $\Gamma$; mais elles n'interviendront que lorsque $\Gamma$ sera positif (c'est à dire que chacun des deux points sera intérieur au conoïde caractéristique issu de l'autre) et il est entendu désormais qu'elles seront réputées nulles dans le cas contraire.

Moyennant la formation de la quantité $v$ si $m$ est impair, des quantités $\dot{V}$ et $\mathcal{V}$ si $m$ est pair, les formules mentionnées ci-dessus résolvent le problème de Canchy, c'est à dire font connaître la valeur de $u_{0}$ lorsqu'on donne $u_{2}$ et $u_{2}^{\prime}$ en tous les points de $S_{2}$.

Mais on peut aussi commencer par calculer, à l'aide des mêmes données $u_{2}$ et $u_{2}^{\prime}$, la valeur $u_{1}$ de $u$ en un point quelconque I de $S_{1}$, ainsi que la valeur $u_{1}^{\prime}$ de la dérivée transversale $u_{1}^{\prime}=\frac{d u}{d v_{1}}$ au même point $\left(v_{1}\right.$ désignant la direction transversale à $S_{1}$ ); puis, considérant ces quantités $u_{1}$ et $u_{1}^{\prime}$ comme de nouvelles données de Cauchy, s'en servir pour le calcul de $u_{0}$. Si dans l'expression ainsi obtenue, on remplace $u_{1}$ et $u_{1}^{\prime}$, en chaque point de $S_{1}$, par leurs valears, on aura à nouveau $u_{0}$ exprimé à l'aide des valeurs de $u_{2}$ et de $u_{2}^{\prime}$.

Ces deux modes de calcul doivent conduire au même résultat: c'est en cela que consiste la forme $A$ du principe de Huyghens. Il est clair qu'on obtiendra ainsi des propriétés soit de la solution élémentaire $v$, soit des fonctions $V$ et $\mathscr{V}$ qui y figurent.

Pour $m$ impair, on aura ainsi l'expression de $v_{02}$ (solution élémentaire formée ${ }^{1}$ avec le point o et un point de $S_{2}$ ) à l'aide des valeurs de $v_{01}, v_{12}$ de cette même solution élémentaire, ainsi que des valeurs $v_{01}^{\prime}, v_{12}^{\prime}$ de la dérivée transversale aux

$1 v$ est solution de l'équation donnée par rapport aux coordonnées du premier point mentionné en indice, et de l'équation adjointe par rapport aux coordonnées du second point. 
différents points de $S_{1}$. C'est ce calcul que je me suis proposé d'exécuter dans le travail cité $(P)$. Il se présente, dans ce cas de $m$ impair, sous une forme particulièrement simple, grâce à l'intervention du symbole particulier d'intégration qu'introduit, dans ce cas, la résolution du problème de Cauchy. Il arrive en effet que, dans les différentiations d'intégrales auxquelles on est conduit, la présence de ce symbole dispense $(A M, \mathbf{6} b i s, \mathbf{1 4} ; Y, 87,93)$ de considérer les termes frontières.

Mais précisément en raison de cette circonstance, l'interprétation des résultats obtenus est moins claire et moins instructive. C'est dans le cas opposé qu'il convient de se placer tout d'abord pour discuter non seulement l'allure des résultats obtenus à la frontière de $S_{2}$, mais aussi ce qui se passe au niveau de ce que nous appellerons, un peu plus loin, le front interne.

Le désir de m'associer à l'hommage que la Science rend à M. Mittag-Leffler me fait affronter aujourd'hui cette dernière partie du calcul, dont la complication relative m'avait fait hésiter jusqu'à présent. Je m'excuse vis à vis du lecteur d'abord de cette complication même à laquelle je n'ai pu échapper complètement, ensuite des fautes qu'elle peut avoir entrainées et que je serais reconnaissant aux géomètres de me signaler. J'espère toutefois les avoir, d'une manière générale, évitées grâce à l'aide qu'un de mes meilleurs collègues et amis a bien voulu m'apporter sur ce point et pour laquelle je tiens à lui exprimer mes bien chaleureux remerciements.

I.

1. Le nombre des différentiations et, par conséquent, leur difficulté augmente avec la valeur de $m$ : je me placerai uniquement dans le cas le plus simple et, d'ailleurs le plus important au point de vue des applications physiques, celui de $m=4$. La formule de résolution s'écrit alors

(1)

$$
\begin{aligned}
& \left(2 \pi u_{0}=-\operatorname{SSS}_{T} \mathcal{V} \varphi d T+\operatorname{SS}_{S}\left[u\left(\frac{d \mathcal{V}}{d \nu}-L \mathcal{V}\right)-u^{\prime} \mathcal{V}\right] d S\right. \\
& +\operatorname{SS}_{\Gamma} V \varphi \frac{d T}{d \gamma}-\mathrm{S}_{\sigma}\left[u\left(\frac{d V}{d \nu}-L V\right)-u^{\prime} V\right] \frac{d S}{d \gamma} \\
& +\mathrm{S}_{\sigma} u \mathcal{V} \frac{d I}{d \nu} \frac{d S}{d \gamma} \\
& +\frac{d}{d \gamma} \underset{\sigma^{\prime}}{\mathbf{S}} u V \frac{d \Gamma}{d \nu} \frac{d S}{d \gamma},
\end{aligned}
$$


Le principe de Fuyghens dans le cas de quatre variables indépendantes. 207 ( $u^{\prime}$ étant la dérivée transversale de $u$ en un point quelconque de $S$ ): soit, en désignant par une notation spéciale chaque terme

$$
2 \pi u_{0}=(a)+(b)+(c)+(d)+(e)+(f)+(g)
$$

$\operatorname{arec}^{1}$

$$
\left\{\begin{array}{c}
(a)=-\iiint \int_{T} \mathcal{V}_{\varphi} d T,(b)=\iiint_{S^{\prime}} u\left(\frac{d \mathcal{V}}{d \nu}-L \mathcal{V}\right) d S,(c)=-\iiint_{S} u^{\prime} \mathcal{V} d S, \\
(d)=\iiint_{I^{\prime}} V_{\varphi} \frac{d T}{d \gamma},(e)=-\iint_{\sigma} u\left(\frac{d V}{d \nu}-L V-\mathcal{V} \frac{d \Gamma}{d \nu}\right) \frac{d S}{d \gamma},(f)=\iint_{\sigma} u^{\prime} V^{d} \frac{d S}{d \gamma}, \\
(g)=\frac{d}{d \gamma} \iint_{\sigma^{\prime}} u V^{\frac{d}{d}} \frac{d}{d \nu} \frac{S}{d \gamma} .
\end{array}\right.
$$

Dans cette formule ${ }^{1}, d T$ désigne l'élément de volume $d x_{1} d x_{2} \ldots d x_{m}$ d'espace à $m$ dimensions, l'intégration correspondante (terme (a)) étant étendue à la portion d'espace limitée par la multiplicité $S$ qui porte les données de Cauchy et par le conoïde caractéristique ayant pour sommet le point $0 ; d S$, un élément superficiel de $S$, - élément évalué d'une manière quelconque, sous la condition ${ }^{2}$ de choisir en conséquence le facteur de proportionnalité qui figure dans les paramètres directeurs $\pi_{1}, \ldots, \pi_{m}$ de l'hyperplan tangent à $S$ et, par conséquent, dans

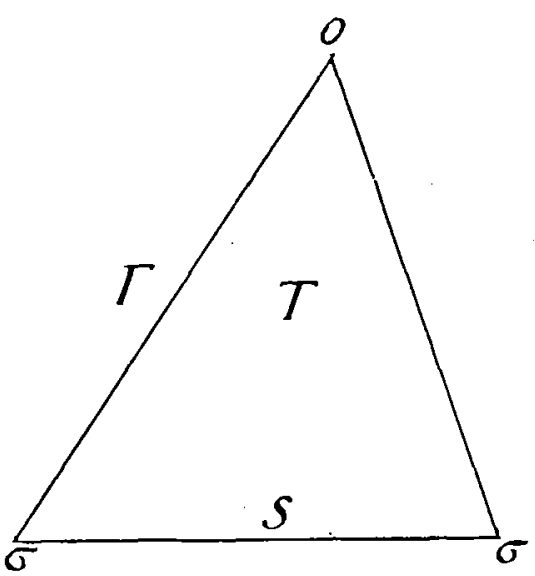

Fig. 1 . la différentielle transversale $\frac{d}{d v}$ : l'intégration correspondante (termes $\left.(b),(c)\right)$ est étendue à la portion $\bar{S}$ de $S$ qui est comprise à l'intérieur du conoïde caractéristique.

Dans les termes $(d),(e),(f),(g)$ figurent les symboles différentiels $\frac{d T}{d \gamma}, \frac{d S}{d \gamma}$

${ }^{1} A M, 35 ; Y, 145$. Deux termes de la formule gènérale ont pu être réunis en un seul (terme (e)) pour la valeur particulière $m=4$ que nous considérons, alors que, pour $m>4$, ils seraient distinets, le premier comportant une différentiation d'ordre $m / 2-2$.

$2 Y, n^{\circ} 39$. 
définis précédemment $(A M$, p. $375 ; Y, 39)$ : le premier, par exemple, représente un élément de surface du conoïde caractéristique, choisi de telle façon que, multiplié par $d \gamma$, il donne un élément du volume compris entre ce conoïde $\Gamma=0$ et la surface voisine $\Gamma=$ const. $=d \gamma$. Si, par exemple, $d \Sigma$ est l'élément superficiel du conoìde calculé à la manière ordinaire et $\frac{d}{d n}$ une dérivation normale ordinaire, on aura

$$
\frac{d T}{d \gamma}=d \Sigma:\left(\frac{d \Gamma}{d n}\right)
$$

Comme nous y conduit naturellement cette formule, nous considérons l'expression (3) comme susceptible de signe: un côté positif est choisi par rapport à $\Gamma=\mathrm{o}$ (ou, dans le terme $(g)$, à $\Gamma=$ const.) et le signe de l'expression précédente sera celui du dénominateur $\frac{d \Gamma}{d n}$. Dans les formules (2), le signe est partout + , c'est à dire que le côté positif choisi est celui des $\Gamma$ croissants.

On peut encore dire que si $\lambda_{1}, \ldots, \lambda_{m-1}$ sont des coordonnées curvilignes quelconques sur $\Gamma$, de telle manière que $\lambda_{1}, \ldots, \lambda_{m-1}, \Gamma$ représentent des coordonnées curvilignes dans l'hyperespace, on aura

$$
\frac{d T}{d \gamma}=J d \lambda_{1} d \lambda_{2} \ldots d \lambda_{m-1}
$$

$J$ désignant le jacobien de $x_{1}, x_{2}, \ldots x_{m}$ par rapport aux coordonnées curvilignes en question rangées de telle façon que le $m^{\text {dare }}$ formé par les directions correspondantes et le sens positif pris sur $n$ soit direct. Le symbole $\frac{d S}{d \gamma}$ se définit d'une manière toute semblable. L'intégration triple $(d)$ s'étend à la surface du conoïde limitée à $S$; l'intégration double $(e)$, à l'intersection $\sigma$ du conoïde et de $S$. Quant à l'intégrale $\iint u V \frac{d \Gamma}{d \nu} \frac{d S}{d \gamma}$, qui doit être différentiée par rapport au paramètre arbitraire $\gamma$ pour obtenir le terme $(f)$ (le paramètre recevant finalement la valeur o après le différentiation), elle est étendue à la multiplicité $\sigma^{\prime}$, intersection de $S$ avec l'hypersurface $\Gamma=\gamma$ que nous appellerons »conoïde modifié», le symbole $\frac{d S}{d \gamma}$ étant défini sur cette dernière comme sur le conoïde lui-même.

Les symboles SSS, SS, S, introduits dans la théorie générale pour le cas d'un nombre $m$ de dimensions arbitraire, représentent ici respectivement des 
Le principe de Huyghens dans le cas de quatre variables indépendantes. 209 symboles d'intégration quadruple, triple et double par lesquels nous les remplaçons: cela est d'ailleurs nécessaire, car nous aurons à envisager des intégrations simples, pour lesquelles notre notation précédente ne fournirait pas de signe.

2. Commençons maintenant par présenter des remarques géométriques de deux sortes.

La première concerne l'application du symbole différentiel ci-dessus considéré à la différentiation d'intégrales multiples. Si, dans une telle intégrale, - par exemple une intégrale SSS étendue à une portion de l'hyperespace, - la forme $d u$ domäine d'intégration varie avec le paramètre $\gamma$ par rapport auquel on différentie; on doit adjoindre au résultat de la différentiation sous le signe intégral un terme de frontière. Or, celui-ci (Cf. $Y, 147$ ) s'exprime avec la plus grande simplicité à l'aide de notre symbole différentiel: l'élément d'intégrale frontière à introduire ne sera autre (pour l'intégrale $m^{\text {uple }}$, par exemple) que

$$
\operatorname{SS} \varphi \frac{d T}{d \gamma}
$$

$f\left(x_{1}, x_{2}, \ldots x_{m}\right)$ étant la quantité sous le signe SSS dans l'intégrale donnée et le côté positif adopté, conformément à ce qui précède, par rapport à la frontière étant le côté extérieur au domaine d'intégration.

Cette expression est valable même dans le cas où, indépendamment de la frontière variable, le domaine d'intégration comporte une frontière fixe rencontrant la première: il n'y aura pas de terme supplémentaire relatif à cette intersection.

Nous aurons besoin de différentier des intégrales de ce genre, et par conséquent, d'écrire le symbole $\frac{d T}{d \gamma}$, dans le cas où l'équation de la frontière variable sera de la forme

$$
\psi(x, \gamma)=0
$$

au lieu d'être résolue par rapport au paramètre. En appliquant à la dérivée normale qui figure dans la formule (3) la règle de dérivation des fonctions implicites, on trouve immédiatement

$$
\frac{d T}{d \gamma}=-\frac{d T}{d \psi} \frac{\partial \psi}{\partial \gamma}
$$

Des termes analogues se présenteront, bien entendu, - et se calculeront de même si, à l'intérieur du domaine d'intégration se présente une cloison au passage de laquelle la quantité à intégrer subit une discontinuité de première 27-2661. Acta mathematica. 49. Imprimé le 6 juillet 1926. 
espèce, et que la position de cette cloison varie avec le paramètre par rapport auquel on doit différentier. L'élément d'intégrale analogue à celui de (5) contiendra alors en facteur, au lieu de la valeur de $f$, celle du saut brusque que fait cette fonction.

2 bis. Le symbole $\frac{d T}{d \gamma}$ comporte une généralisation évidente à une intégration étendue à l'intersection (supposée orientée) des deux multiplicités $F_{1}\left(x_{1}, x_{2}, \ldots x_{m}\right)=0$, $F_{2}\left(x_{1}, x_{2}, \ldots x_{m}\right)=0$, ces deux hypersurfaces étant supposées non tangentes entre elles. Considérant chacune d'elles comme faisant partie d'une famille $F_{1}=\gamma_{1}$ ou $F_{2}=\gamma_{2}$, on pourra définir le symbole

$$
\frac{d T}{d \gamma_{1} d \gamma_{2}}
$$

comme égal à $J d \lambda_{1} d \lambda_{2} \ldots d \lambda_{m-2}$, en désignant par $\lambda_{1}, \lambda_{2}, \ldots, \lambda_{m-2}$ des coordonnées curvilignes sur l'intersection des deux surfaces, et par $J$ le jacobien des $x$ par rapport à $\lambda_{1}, \lambda_{2}, \ldots, \lambda_{m-2}, \gamma_{1}, \gamma_{2}$. Ce nouveau symbole interviendra évidemment dans le calcul de la dérivée seconde $\frac{\partial^{2} I}{\partial \gamma_{1} \partial \gamma_{2}}, I$ étant une intégrale étendue à un domaine dont la frontière est constituée totalement ou partiellement par des portions d'hypersurfaces $F_{1}=\gamma_{1}, F_{2}=\gamma_{2}$.

Dans le cas opposé où les hypersurfaces $F_{1}=0, F_{2}=0$ sont tangentes, il nous sera utile de remarquer que, les différentielles $d F_{1}$ et $d F_{2}$ étant proportionnelles, le symbole $d F_{2} / d \dot{F}_{1}$ a un sens au point de contact, indépendamment du chemin suivi pour aboutir en ce point (sous la condition qu'il ne soit pas tangent aux hypersurfaces).

3. Les remarques qui viennent d'être présentées sur la différentiation des intégrales permettent $(Y, 147)$ d'écrire sous une autre forme les termes $(d),(e)$; $(f),(g)$ de notre formule $(2)$, savoir ${ }^{1}$

$$
\left\{\begin{array}{c}
(d)=-\frac{d}{d \gamma} \iiint \int V_{\varphi} d T,(e)=\frac{d}{d \gamma} \iiint u\left(\frac{d V}{d \nu}-L V-\mathcal{V}^{\frac{d}{d \nu}}\right) d S \\
(f)=-\frac{d}{d \gamma} \iiint u^{\prime} V d S \\
(g)=-\frac{d^{2}}{d \gamma^{2}} \iiint u V \frac{d \Gamma}{d \nu} d S
\end{array}\right.
$$

1 On observera que, dans ces formules $\left(2^{\prime}\right)$, les signes (voir ceux qui figurent devant $(d),(f)$ ) sont opposés à ceux qui figuraient au passage correspondant de $Y$ (no 147, formule (30)): 
Le principe de Huyghens dans le cas de quatre variables indépendantes. 211

les intégrales soumises à la différentiation par rapport à $\gamma$ étant étendues, pour $(d)$, à l'intérieur du »conoïde modifié» $\Gamma=\gamma$, c'est à dire à la portion de $T$ qui satisfait à l'inégalité $\Gamma \geq \gamma$; pour $(e),(f),(g)$, à la portion de $S$ qui satisfait à la même inégalité, et le paramètre $\gamma$ devant recevoir la valeur zéro après différentiation. .

Les deux formes nous seront utiles, ainsi qu'il apparaitra plus loin.

4. Considérons maintenant, dans l'espace ordinaire, deux surfaces, l'une fixe $\Sigma$ que nous supposerons tangente au plan des $x y$ à l'origine des coordonnées; l'autre variable $\Sigma^{\prime}$ et dépendant d'un paramètre $\gamma$. Pour la valeur $\gamma=0, \Sigma^{\prime}$ sera également tangente au plan des $x y$ à l'origine et, de plus, située, par rapport à $\Sigma$, du côté des $z$ négatifs, de telle sorte que la différence $z-z^{\prime}$ des cotes des deux surfaces sera positive. Il sera même supposé que, dans tout le voisinage de l'origine, cette différence sera un infiniment petit du second ordre (et jamais d'ordre supérieur) par rapport à $\varrho=\sqrt{x^{2}+y^{2}}$ considéré comme infiniment petit principal. Par contre, $z^{\prime}$ sera une fonction croissante de $\gamma$; de sorte que, pour $\gamma>0$, les deux surfaces se couperont mutuellement suivant deux petites calottes délimitant entre elles un petit espace lenticulaire. Nous envisagerons une intégrale de surface, telle que

$$
I=\iint h d \Sigma
$$

étendue à l'une de ces calottes, la seconde par exemple, et qui pourra d'ailleurs s'écrire

$$
I=\iint I(x, y, \gamma) d x d y
$$

Les deux surfaces seront supposées soit analytiques, soit tout au moins "régulières», c'est à dire que $z, z^{\prime}$ seront supposés développables, l'un en fonction de $x, y$, l'autre en fonction de $x, y, \gamma$, soit en séries de Maclaurin (cas analytique), soit, du moins, par la formule de Maclaurin poussée jusqu'aux termes d'un certain ordre, supposé suffisant pour les considérations ultérieures. L'hypothèse correspondante sera faite sur la fonction qui figure dans la formule (7).

La valeur $\gamma=0$ est, pour les divers éléments relatifs à l'intersection des deux surfaces, une valeur critique et il est évident que plusieurs d'entre eux

i cet endroit, en effet, les intégrales soumises à la différentiation étaient supposées relatives aux domaines définis par les inégalités $o \leq \Gamma \leq \gamma$, c’est à dire à l'extérieur du conoïde modifié. 
donnent lieu à des développements ordonnés non suivant les puissances entières de $\gamma$, mais suivant celles de $\sqrt{\gamma}$; et l'intersection devient même imaginaire, dans les hypothèses indiquées, pour $\gamma<0$. Cependant, si l'on prend d'abord l'exemple le plus simple, celui de l'aire $\Sigma$ d'une calotte découpée sur une sphère par une autre sphère presque tangente à la première, $\gamma$ désignant alors le segment très petit de ligne des centres intercepté entre les deux surfaces, il apparait sans difficulté que $\Sigma$ est développable en série entière par rapport à $\gamma$.

Ce résultat est général: nous allons voir qu'il s'applique, moyennant les hypothèses précédentes, au problème tel que nous l'avons posé, à ceci près, bien entendu, que le développement n'existe que jusqu'à un certain ordre lorsqu'il en est ainsi pour les fonctions qui figurent dans les données de la question.

Pour le démontrer, considérons d'abord, sur $\Sigma^{\prime}$, le point de contact d'un plan tangent parallèle au plan des $x y^{1}$ et supposons, pour simplifier, que le lieu de ce point, lorsque $\gamma$ varie, soit l'axe des $z$, sa troisième coordonnée $\zeta$ admettant un développement (limité à un certain ordre ou indéfini) suivant les puissances entières de $\gamma$. Il sera commode d'ailleurs, de considérer $\gamma$ et $\zeta$ comme deux variables indépendantes jusqu'à la fin du calcul, où nous remplacerons $\zeta$ par sa valeur. L'équation de la surface $\Sigma^{\prime}$ sera alors de la forme

$$
z^{\prime}=\zeta-z_{2}^{\prime}(x, y)-z_{3}^{\prime}(x, y)-\cdots
$$

les différents termes étant des polynômes homogènes en $x, y$ de degrés marqués par leurs indices; celle de $\Sigma$ sera

$$
z=z_{2}(x, y)+z_{3}(x, y)+\cdots
$$

d'où

$$
z-z^{\prime}=-\zeta+Z_{2}(x, y)+Z_{3}(x, y)+\cdots \quad\left(Z_{i}=z_{i}+z_{i}^{\prime}\right)
$$

D'après l'hypothèse faite sur la position relative des deux surfaces pour $\gamma=0$, l'ensemble $Z_{2}(x, y)=A x^{2}+2 B x y+C y^{2}$ des termes du second ordre de $z-z^{\prime}$

${ }^{2}$ Les équations $\frac{\partial z^{\prime}}{\partial x}=0, \frac{\partial z^{\prime}}{\partial y}=0$ qui font connaitre le point de contact en question ont un jacobien différent de zéro si l'origine des coordonnées n'est pas, pour la position initiale de $\Sigma^{\prime}$, un point parabolique, ce qu'on peut toujours supposer moyennant une transformation ponctuelle effectuée sur tonte la figure (et consistant par exemple à ajouter, tant à $z$ qu'à $z^{\prime}$ un polynôme du second degré en $x, y$ ). 
Le principe de Huyghens dans le cas de quatre variables indépendantes.

213

constitue une forme définie positive. Dans ces formules, nous ferons $\zeta=\varepsilon^{2}$ et, d'autre part, nous introduirons, dans le plan des $x y$, des coordonnées polaires $\varrho, \theta, Z_{i}(\theta)$ étant mis d'une manière générale pour $Z_{i}(\cos \theta, \sin \theta)$, on aura ainsi

$$
z-z^{\prime}=-\varepsilon^{2}+\varrho^{2} Z_{2}(\theta)+\varrho^{3} Z_{3}(\theta)+\cdots
$$

le coefficient $Z_{2}(\theta)$ ayant la valeur $A \cos ^{2} \theta+2 B \cos \theta \sin \theta+C \sin ^{2} \theta$.

Notons que le second membre de cette équation ne doit pas changer par le changement simultané de $\varrho$ en $-\varrho$ et de $\theta$ en $\theta+\pi$. Les coefficients des puissances impaires de $\varrho$ sont donc »imparisymétriques» en $\theta$, c'est à dire changent de signe par le changement de $\theta$ en $\theta+\pi$ (leurs développements trigonométriques ne contenant, par conséquent, que des multiples impairs de $\theta$ ), tandis que les coefficients des puissances paires seront "parisymétriques», c'est à dire développables par rapport aux lignes trigonométriques de multiples de $2 \theta$. Pour abréger le langage, convenons de dire qu'une expression est "paire en $\varrho, \theta$ " ou "impaire en $\varrho, \theta$ », suivant qu'elle reste inaltérée par le changement simultané de $\varrho$ en $-\varrho$ et de $\theta$ en $\theta+\pi$, ou qu'elle change de signe dans ces conditions.

L'intersection des deux surfaces s'obtient en égalant à zéro le second membre de $\left(8^{\prime}\right)$. Moyennant l'introduction de notre variable $\varepsilon$, on peut extraire les racines carrées et écrire

$$
\frac{\varepsilon}{\sqrt{Z_{2}(\theta)}}=\varrho\left[\mathrm{I}+\varrho T_{3}(\theta)+\cdots\right]
$$

Le facteur de $\varrho$ est, d'après la manière dont il est formé, pair en $\varrho, \theta$, de sorte que $\varepsilon$ est impair par rapport aux mêmes quantités. Si donc on résoud en $\varrho$ cette équation, ce qui est possible puisque $Z_{2}$ est différent de zéro pour toute valeur réelle de $\theta$, soit

$$
\varrho=\frac{\varepsilon}{\sqrt{Z_{2}(\theta)}}+V_{3}(\theta)\left(\frac{\varepsilon}{\sqrt{Z_{2}}}\right)^{2}+\cdots
$$

le second membre sera lui-même impair en $\varepsilon, \theta$.

La quantité $H$ qui figure dans l'intégrale (7) s'écrit, d'autre part,

$$
H=H_{0}+\varrho H_{1}(\theta)+\varrho^{2} H_{2}(\theta)+\cdots,
$$

les coefficients des diverses puissances de $\varrho$ étant, en $\theta$, de »parisymétries» correspondant à la parité de leurs indices. L'intégrale double sera 


$$
\iint\left(H_{0}+\varrho H_{1}+\varrho^{2} H_{2}+\cdots\right) \varrho d \varrho d \theta=\int_{0}^{2 \pi} d \theta\left(H_{0} \varrho_{2}^{2}+H_{1} \varrho_{3}^{3}+\cdots\right) .
$$

Le domaine d'intégration étant l'aire intérieure à l'intersection des deux surfaces projetées sur le plan des $x y$, on doit, dans l'intégrale simple du second membre, remplacer $\varrho$ par sa valeur (9), ce qui donne, sous le signe $\int$, un développement ordonné suivant les puissances de $\varepsilon$ et pair en $\varepsilon, \theta$.

Or, toute quantité imparisymétrique en $\theta$ donne, entre $O$ et $2 \pi$, une valeur moyenne nulle. Donc il ne restera que les puissances paires de $\varepsilon$, c'est à dire les puissances entières de $\zeta$, et le résultat, une fois $\zeta$ remplacé par sa valeur, sera une série entière en $\gamma$, ainsi que nous voulions le démontrer.

Le premier terme. du développement de l'intégrale suivant les puissances de $\gamma$ donnera la dérivée correspondante $\frac{d I}{d \gamma}$ (laquelle est fournie par la méthode précédemment indiquée, avec introduction du symbole $\frac{d \Sigma}{d \gamma}$, pour $\gamma>0$, mais non pour $\gamma=0$ ). Dans ce premier terme, la valeur de $H$ à l'origine sera en facteur, multipliée par $\frac{d \zeta}{d \gamma}$ et par la quantité (nécessairement différente de zéro, dans nos hypothèses)

$$
\frac{\mathrm{I}}{2} \int_{0}^{2 \pi} \frac{d \theta}{Z_{2}(\theta)}=\frac{\mathrm{I}}{2} \int_{0}^{2 \pi} \frac{d \theta}{A \cos ^{2} \theta+2 B \cos \theta \sin \theta+C \sin ^{2} \theta}=\frac{\pi}{\sqrt{A C-B^{2}}}
$$

Quant à l'hypothèse que le lieu $d u$ point de contact de $\Sigma^{\prime}$ avec son plan tangent parallèle au plan des $x y$ est l'axe des $z$, elle ne diminue pas la généralité, du moins si l'on suppose que ce lieu admet l'origine comme point ordinaire et n'est pas tangent lui-même au plan des $x y$ : s'il en est ainsi, en effet, les deux premières coordonnées $\xi, \eta$ du point en question seront développables suivant les puissances entières de la troisième d'entre elles

$$
\xi=\xi(\zeta), \eta=\eta(\zeta)
$$

et, en effectuant sur la figure et les expressions considérées ci-dessus la transformation ponctuelle 
Le principe de Huyghens dans le cas de quatre variables indépendantes.

$$
x_{1}=x-\xi(z), \quad y_{1}=y-\eta(z), z_{1}=z
$$

on sera ramené au cas précédent.

Le calcul précédent s'étend de lui-même aux cas:

$I^{\circ}$ où les deux surfaces seraient variables, leur disposition relative pour $\gamma=0$ d'une part, pour $\gamma \neq 0$ de l'autre, restant celle que nous venons de considérer;

$2^{\circ}$ où (comme dans la théorie classique du contact) l'une des surfaces, $\boldsymbol{\Sigma}^{\prime}$ celle sur laquelle on intègre, serait rapportée à des coordonnées curvilignes quelconques $X, Y$, l'autre $\boldsymbol{\Sigma}^{\prime}$ étant donnée par son équation (résolue ou non par rapport à l'une des coordonnées cartésiennes). La quantité, analogue à (8), qui, égalée à zéro, donnerait la courbe limite de l'aire d'intégration serait alors le résultat de substitution des coordonnées (cartésiennes) de $\Sigma^{\prime}$ dans l'équation de $\Sigma$, et $\zeta$ désignerait alors la valeur initiale (au point de contact des deux surfaces, pour $\gamma=0$ ) de cette quantité. Les transformations qui nous ont servi ci-dessus (transformation ( $\mathrm{I}$ ) et introduction des coordonnées polaires) seraient à opérer dans le plan des $X Y$.

II.

5. Nous opérerons comme dans $(P)$ en partant de la figure principale (figure 2) de ce Mémoire, laquelle est reproduite ci-dessous avec quelques changements de notation. Le conoïde de sommet o étant coupé successivement suivant les deux sections $S_{1}, S_{2}$, nous désignerons par $T_{1}$ la portion d'espace à quatre dimensions limitée dans le conoïde par la première d'entre elles, par $T_{2}$ la portion comprise, dans l'intérieur du même conoïde, entre les deux sections. ${ }^{1}$ Un point quelconque I de $S_{1}$ sera le sommet d'un nouveau conoïde, lequel découpera dans la multiplicité $S_{8}$ une portion $\overline{\bar{S}}_{2}$ et délimitera avec elle une portion d'hyperespace $\overline{\bar{T}}_{2}$. La frontière de $S_{1}$ sera désignée par $\sigma$; celle de $\bar{S}_{2}$, par $\bar{\sigma}_{2}$. Il y aura également lieu de considérer la portion $\Gamma$ du conoỉde caractéristique primitif comprise entre le sommet et $S_{1}$, la portion $I_{2}^{\prime}$ du même conoïde comprise entre $S_{1}$ et $S_{2}$ et la portion $\bar{\Gamma}_{2}$ du conoïde de sommet I limitée à $\overline{\overline{S_{2}}}$.

Considérées comme lieux d'un second point 2 , l'hypersurface $\bar{\Gamma}_{2}$ et la surface (multiplicité deux fois étendue) $\bar{\sigma}_{2}$ sont définies, dans $T_{2}$ et dans $S_{2}$, par l'équation $\Gamma_{12}=0$, pendant que $\bar{T}_{2}$ et $\overline{S_{2}}$ sont définis par l'inégalité $\Gamma_{12} \geq 0$.

\footnotetext{
${ }^{1}$ Dans le Mémoire précédent $(H)$, ce que nous appelons $T_{2}^{\prime}$ était désigné par $T_{2}-T_{1}$.
} 
Si maintenant nous nous donnons au contraire, le point 2 (dans $T_{2}$ ou sur $S_{\mathrm{z}}$ ) en considérant le point 1 comme variable sur $S_{1}$, l'inégalité $\Gamma_{12} \geq 0$ définira une portion de $S_{1}$ que nous désignerons ${ }^{1}$ par $\underline{S}_{1}$, pendant que la nappe conoïdale
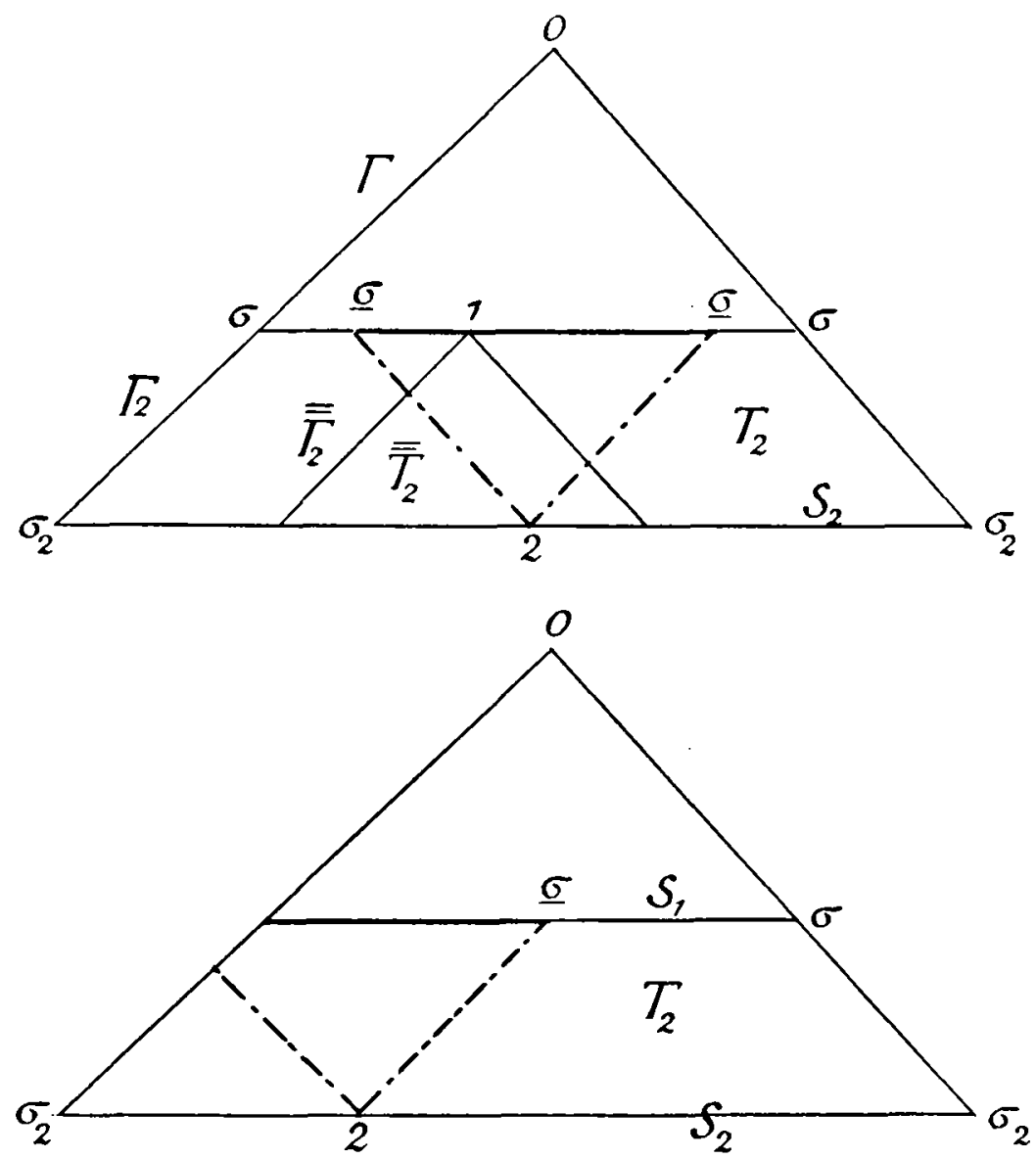

Fig. 2.

de sommet 2 , définie par l'équation $\Gamma_{19}=0$, coupera $S_{1}$ suivant une trace ${ }^{1}$ que nous désignerons par $\underline{\sigma}$.

Sur nos figures 2, 2 bis, ainsi que sur certaines des figures qui suivront

${ }^{1}$ Contrairement aux notations de $P$, il est entendu que $S_{1}$ et $S_{2}$ sont limitées a l'intérieur du conoïde de sommet $o$. Il en est, par conséquent, de même pour $\underline{S}_{1}$ et $\underline{\sigma}$. Ajoutons que l'indice I accompagnant les lettres $S, \sigma, \lambda$ sera fréquemment omis dans ce qui va suivre, ancune confusion n'étant à craindre de ce chef: les intégrations, dans la formule finale (14) (no 19), et les intégrations intérieures dans les formules définitivement obtenues par chacun des calculs des $n^{\text {os }}$ 10-18, sont relatives à des domaines situés au $S_{1}$, sans qu'il soit utile de le spécifier chaque fois. 
Le principe de Huyghens dans le cas de quatre variables indépendantes.

(fig. $b a_{1}, b d_{1}$, ete.), ces diverses variétés sont représentées schématiquement, chacune d'elles y figurant avec deux dimensions de moins qu'elle n'en a en fait.

5 bis. Enfin, l'intervention des termes $(g)$ et, en vertu de $\left(2^{\prime}\right)$, celle des termes $(d),(e),(f)$ nous conduira à considérer:

l'équation $\Gamma_{01}=\gamma_{1}, \gamma_{1}$ désignant une constante très petite ${ }^{1}$;

l'équation $\Gamma_{12}=\gamma_{2}, \gamma_{2}$ désig-

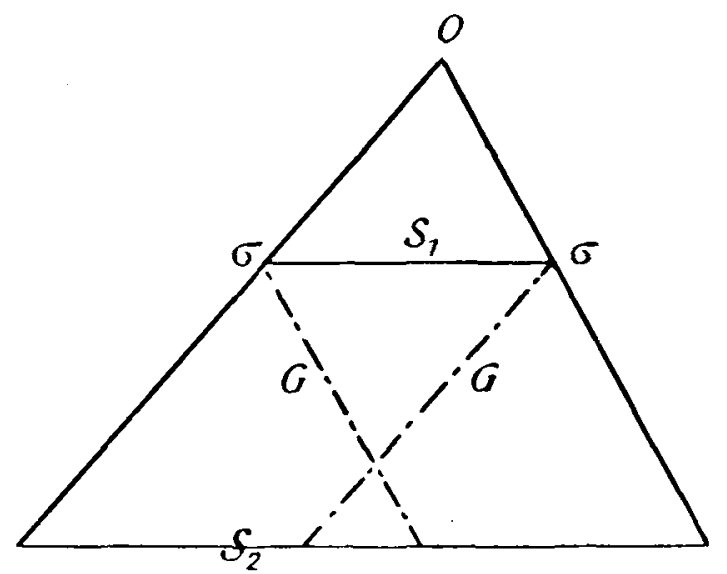

Fig. 2 bis. nant une seconde constante arbitraire très petite;

l'inégalité $\Gamma_{12} \geq \gamma_{2}$.

La première équation définit, sur $S_{1}$, une variété à deux dimensions $\sigma^{\prime}$ (trace du »conoïde modifié» $\Gamma_{0 \mathrm{t}}=\gamma_{1}$ ) à laquelle est étendue l'intégrale $(g)$, portant sur $u$. L'équation $\Gamma_{12}=\gamma_{q}$, lorsque le point I est donné, donne comme lieu du point 2 , une conoïde modifié $\overline{\bar{l}}, 2$ (figures $b d_{1}, c d_{1}$ ) dont la trace sur $S_{2}$ sera désignée par $\overline{\bar{\sigma}}_{, 2} ;$ l'inégalité $\Gamma_{12} \geq \gamma_{2}$, une portion de $T_{2}$ qui sera désignée par $\bar{T}_{12}$. $\mathrm{Si}$ e'est au contraire le point 2 qui est donné, le lieu défini par l'équation $\Gamma_{12}=\gamma_{2}$ sur $S_{1}$ sera dénommé $\sigma_{1}$; la portion de $S_{1}$ intérieure à ce lieu, autrement dit définie par l'inégalité $\Gamma_{12} \geq \gamma_{2}$, sera désignée par $\underline{S}_{11}$ ou plus simplement ${ }^{2} S$,

6. L'inspection de la figure 2 montre immédiatement que, suivant la position du point 2 , divers cas sont possibles en ce qui regarde la situation relative de $S_{1}$ par rapport à $S_{1}$. On y distingue, tant dans $T_{2}$ que sur $S_{2}$, deux régions, l'une centrale $\left(T_{2 c}, S_{2 c}\right)$, l'autre annulaire $\left(T_{2 a}, S_{2 a}\right)$ : la première est celle où doit être le point 2 pour que $S_{1}$ soit entièrement intérieur à $S_{1}$; la seconde est constituée par les points 2 dont le conoïde caractéristique coupe la frontière $\sigma$ de $S_{1}$. La frontière entre la région centrale $T_{2 c}$ et la région annulaire $T_{2 a}$ est le lieu des points tels que les traces $\sigma$ et $\sigma$ des conoïdes caractéristiques de sommets $o$ et 2 soient tangentes entre elles intérieurement: cette hypersurface $G$ n'est autre que la seconde nappe (située dans $T_{2}$ ) de l'enveloppe des conoïdes caractéristiques ayant leurs sommets sur $\sigma$, la première nappe étant constituée par le conoïde

1 Pour alléger la notation, nous désignerons par $\gamma_{1}, \gamma_{2}$ et (au no 20) $\gamma$, les paramètres infinitésimaux qui devraient, logiquement, s'appeler $\gamma_{01}, \gamma_{12}, \gamma_{02}$.

2 Voir la note de la page précédente.

28-2661. Acta mathematica. 49. Imprimó le 6 juillet 1926. 
primitif lui-même, lequel, dans sa portion intérieure à $T_{2}$, bien entendu, est le lieu des points 2 tels que les traces $\sigma$ et $\sigma$ soient tangentes extérieurement. La trace de $G \operatorname{sur} S_{2}$, frontière entre $S_{2 c}$ et $S_{2 \alpha}$, sera désignée par $s_{2}$ et s'appellera le front interne.

Ce front interne joue un rôle important dans les discussions auxquelles le principe de Huyghens à donné lieu. Je fais surtout allusion à la célèbre polémique entre Fresnel et Poisson et qui concernait surtout, comme on sait, ce que j'ai appelé précédemment" la forme $B$ du principe ou encore la »mineure» de Huyghens. L'une des principales objections élevée par Poisson contre cette mineure, - c'est à dire contre le fait qu'une onde sphérique engendrés par un ébranlement produit au voisinage d'un point unique de l'espace et d'un instant unique ne produit d'effet que sur son front même et non en arrière de ce front -, est que cette circonstance, supposée réalisée à un certain instant $t$, devrait cesser de l'être aux instants suivants: il semble, en effet, que la perturbation, localisée (par hypothèse) à l'instant $t$ uniquement sur la surface d'une sphère de rayon $c t$ (en appelant $c$ la vitesse de propagation), devrait engendrer, en même temps que l'onde externe concentrique à $\sigma$ et de rayon croissant avec le temps, une onde rétrograde interne également concentrique à $\sigma$, mais revenant vers le centre avec une vitesse également égale à $c$; et c'est ce phénomène qui se produirait effectivement si la perturbation produite à l'instant $t$ sur $S_{1}$ était quelconque, ainsi que l'avait fort bien vu Poisson.

"La production d'une nouvelle onde en avant de celle que vous considérez», écrit-il à Fresnel ${ }^{2}$, «et la non-communication du mouvement en arrière, n'ont lieu qu'à raison d'un rapport déterminé qui subsiste, dans l'onde donnée, entre les condensations et les vitesses propres des molécules fluides».

Ce rapport déterminé qu'indique Poisson, n'est autre chose, au fond, que le système des conditions de compatibilité telles que les vise la théorie d'Hugoniot: conditions qui sont nécessairement vérifiées par le fait que la perturbation dont il s'agit est engendrée par une onde unique venant du centre initial et non par la rencontre de deux fronts d'ondes venant coincider momentanément suivant $\sigma$ : autrement dit, qu'il n'existait pas, avant l'instant $t$, d'onde centripète venant vers $\sigma$ de l'extérieur.

$1 C$, parties I et IV.

2 Lettre à Fresnel, insérée aux Annales de Chimie et de Physique, t. XXII, p. 270, mars 1823; Oeurres de Fresnel, $3^{\mathbf{e}}$ section, tome II, p. 209. 
On sait comment Riemann, Christoffel et surtout Hugoniot ont obtenu ces conditions, ou plutôt les premières d'entre elles, celles qui expriment qu'une discontinuité d'ordre ${ }^{1} p$ n'est pas destinée à se dédoubler en deux ou plusieurs discontinuités d'ordre $p$. Aux conditions ainsi formées, il faudrait en adjoindre d'autres d'ordre supérieur, exprimant qu'avant ou après l'instant considéré, la discontinuité d'ordre $p$ ne se dédouble pas en une discontinuité d'ordre $p$ et une ou plusieurs discontinuités d'ordre $p+k$ (avec $k>0$ ) (ou même en plusieurs discontinuités d'ordre $p+k)$. Nous avons essayé ${ }^{2}$ d'écrire les plus simples parmi ces conditions d'ordre supérieur; mais elles se compliquent rapidement à mesure que $k$ augmente, et il y a lieu de rechercher, non toutes ces conditions successives, mais la condition totale dont l'ensemble de leurs premiers membres fournit le développement.

Le problème dont nous nous occupons en ce moment introduit et permet, comme j'espère le montrer dans un travail ultérieur, de trouver ces conditions de compatibilité totales, du moins pour les mouvements régis par une équation (E).

Là où elles ne seront pas vérifiées, un front interne, celui dont l'existence est suggérée par Poisson, se produira en même temps que l'onde centrifuge. D'ailleurs, contrairement à ce que pensaient Poisson et Fresnel lui-même, la difficulté dont il s'agit n'est nullement liée à la mineure de Huyghens: la même circonstance paradoxale semble au premier abord se produire, que cette mineure

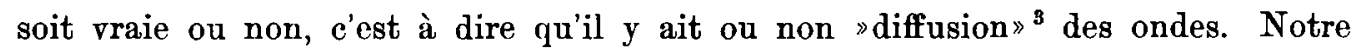
figure 2 permet d'ailleurs de concevoir la disposition de ces deux fronts centrifuge et centripète. Il suffit d'y concevoir le sens de haut en bas comme étant celui des temps croissants, la nappe de conoïde de sommet o représentée sur le dessin comme étant une »nappe d'avenir» et l'ensemble du dessin comme schématisant l'effet produit par un ébranlement originaire du point o de l'espacetemps sur les phénomènes ultérieurs relatifs aux différents points de $S_{2}$. Ce point de vue est inverse de celui où nous nous plaçons en général, lequel consiste à considérer le temps comme croissant de bas en haut de la figure, la nappe conoïdale de sommet o représentée sur notre dessin comme une "nappe de passé» et l'ensemble de nos schémas et de nos calculs comme destiné à déterminer l'effet produit en o par des perturbations initialement données le long de $S_{2}$. Mais -

\footnotetext{
1 Voir nos Leçons sur la propagation des ondes, Chap. II.

2 Ibid. \$ 4.

* Voir C, IV.
} 
c'est là une forme du "principe du retour inverse" - il est au fond indifférent de se placer à l'un ou à l'autre de ces deux points de vue: ils sont équivalents; tout conclusion obtenue dans l'un des cas entraine une conclusion exactement correspondante dans l'autre.

Si l'on considère o comme un centre initial d'ébranlement, le reste de la figure comme correspondant à des instants ultérieurs, $S_{1}$ comme figurant l'un d'eux, $\sigma$ représentera le front d'onde engendré à cet instant par l'effet de l'ébranlement initial. $\Gamma_{2}$ représentera le front externe ultérieur, - qui est le front véritable - et $G$ le front interne dont nous venons de parler.

$\mathrm{Si}$, au contraire, nous reprenons notre point de vue habituel en considérant les ébranlements initiaux comme distribués suivant $S_{z}$ et que, plus spécialement, nous en considérions un qui soit localisé au voisinage immédiat d'un point déterminé 2 de $S_{2}$, cet ébranlement particulier engendrera une onde dont la marche sera figurée par le conoïde $\Gamma_{12}$ de sommet 2 et la position à un certain instant par $\sigma$ si l'instant en question est figuré dans l'espace-temps par $S_{1}$. Au delà de $S_{1}$, ce front d'onde engendre encore un front externe et un front interne et la condition pour que le front interne passe précisément au point o de l'espace-temps est que le point 2 ait été choisi sur la trace $S_{2}$ du front interne $G$ engendré par $\sigma$ dans $T_{2}$ (puisque, - autre répercussion du principe du retour inverse la condition qui définit le front interne, celle que les traces $\sigma$ et $\sigma$ soient tangentes entre elles, est symétrique par rapport aux points o et 2). Si à un tel choix du point 2 devait correspondre une onde produisant un effet spécial aux lieux et instants où elle passe et, si par conséquent les points pris sur $G$ agissaient sur le point o autrement que les autres, la formule de résolution précédemment écrite devrait contenir non seulement des intégrales triples étendues à $S_{2}$ et des intégrales doubles étendues à $\sigma_{2}$, mais aussi des intégrales doubles étendues au front interne $s_{2}$.

Pour la même raison que tout à l'heure, il est absurde a priori qu'il en soit ainsi, et, de fait, notre formule de résolution ne contient rien de pareil. En particulier, comme le remarque Poincaré ${ }^{1}$, il est aisé de s'assurer que les termes de cette espèce disparaissent dans le cas classique de l'équation des ondes sphériques. En appliquant un mode de calcul analogue à l'équation générale (E), je m'attendais à trouver des propriétés de la fonction $V$ qui intervient dans la formule de résolu-

'Théorie Mathématique de la Lumière, Chap. III, p. 98. On sait (loc. cit.) que la compensation dont il s'agit n'a lieu que si l'on tient compte simultanément de tous les termes de la formule, e'est à dire à la fois des déplacement initiaux et des vitesses initiales. 
Le principe de Huyghens dans le cas de quatre variables indépendantes.

221

tion. On verra plus loin que cette attente a été déçue et que la destruction mutuelle des termes relatifs au front interne se produirait de même si, dans la formule fondamentale (1), la fonction $V$ était de forme tout à fait quelconque.

7. La disposition de figure que nous venons de considérer n'est pas la seule qui puisse se présenter. Il peut arriver (figure 2 bis) que le front interne se croise lui-même et qu'il y ait dès lors, non seulement, dans $T_{2}$, comme précédemment, des points 2 tels que $\sigma$ soit entièrement intérieure à $\sigma$ et d'autres tels que $\sigma$ et $\sigma$ soient sécantes, mais en outre, dans $T_{2}$ et particulièrement sur $S_{2}$, des points auxquels corresponde une trace d'onde $\underset{\sigma}{\sigma}$ comprenant entièrement $\sigma$ à son intérieur. Quoique cette nouvelle disposition entraine en général, pour le front interne $G$, l'existence des arêtes de rebroussement auxquelles j'ai déjà fait allusion dans $P$ et dont je compte m'occuper plus en détail dans un travail ultérieur, elle n'entrajne, pour le calcul actuel, que des modifications insignifiantes et évidentes par elles-mêmes. Nous nous bornerons, pour fixer les idées, au cas considéré en premier lieu.

8. Nous ne supposerons donc que deux régions différentes, la région centrale et la région annulaire. Les points de la seconde d'entre elles donneront naissance à des traces $\sigma$ qui couperont chacune $\sigma$ suivant une ligne fermée que nous désignerons par $\lambda$.

S'il s'agissait de l'équation des ondes sphériques

$$
\frac{\partial^{2} u}{C^{2}} \frac{\partial^{2} u}{\partial t^{2}}-\frac{\partial^{2} u}{\partial x^{2}}-\frac{\partial^{3} u}{\partial y^{2}}-\frac{\partial^{2}}{\partial z^{2}}=
$$

(ou, plus généralement, d'une équation ayant les mêmes termes du second ordre que l'équation des ondes sphériques), l'hypersurface $S_{1}$ n'étant autre que $t=$ const., $\sigma$ serait une sphère et il en serait de même de $\sigma$ pour tout choix du point 2 dans la région centrale. Au contraire, pour un point 2 pris dans la région annulaire, $\sigma$ serait une calotte sphérique limitée à son cercle d'intersection avec la sphère $\sigma$.

Nous continuerons, dans le cas général, à admettre que les relations qualitatives de $\sigma$ et de $\sigma$ sont les mêmes que dans ce cas particulier et il nous sera même commode d'employer les mots de "sphéroïde» pour la multiplicité $\sigma$ on la multiplicité $\sigma$ qui correspond à un point de la région centrale, de "calotte sphéroïdale» pour la multiplicité $\sigma$ correspondant à un point de la région annulaire, et limitée par conséquent, à son intersection $\lambda$ avec $\sigma$, de même que pour la calotte ainsi interceptée sur $\sigma$ et que nous désignerons par $\bar{\sigma}$. 
Si nous avons substitué à l'un ou à l'autre des conoïdes $\Gamma_{01}, \Gamma_{12}$ un conoïde modifié ( $\mathrm{n}^{\mathrm{os}} 1,5 \mathrm{bis}$ ), une modification correspondante étant faite éventuellement à un conoỉde de sommet 2 considéré comme lieu du point $I$, et si le point 2 est dans la région annulaire, de sorte que les traces $\sigma$ et $\sigma$ se coupent, chacune des deux calottes qui en résultent sera modifiée (même si un seul des paramètres $\gamma_{1}, \gamma_{2}$ est différent de zéro), soit par changement du sphéroïde auquel elle appartiendra, soit par changement du sphéroïde qui la limitera. Outre le trait supérieur ou inférieur dont nous affecterons la lettre $\sigma$ suivant qu'elle représentera la trace de $\Gamma_{01}=$ const. ou celle de $\Gamma_{12}=$ const., nous inscrirons un accent supérieur, si on fait varier $\gamma_{1}$ et un accent inférieur si on fait varier $\gamma_{2}$. Une convention toute semblable sera faite pour une ligne $\lambda$ modifiée. Ainsi $\bar{\sigma}$, désignera la calotte découpée, sur l'intersection de $S_{1}$ avec $I_{01}=0$ par le conoïde modifié $\Gamma_{12}=\gamma_{2}$ construit à l'aide d'un point déterminé 2 quelconque; $\bar{\sigma}^{\prime}$ la calotte délimitée de la même façon sur le sphéroïde $\bar{\sigma}^{\prime}$, trace du conoïde modifié $\Gamma_{01}=\gamma_{1} ; \lambda_{1}^{\prime}$, l'intersection des traces de deux conoïdes modifiés; etc.

8 bis. Enfin, nous aurons à étudier l'ensemble des positions occupées par le conoïde modifié $\overline{\bar{\Gamma}}_{12}$ (d'équation $\Gamma_{12}=\gamma_{2}$ ) lorsqu'on fera varier le point I, en donnant au paramètre $\gamma_{2}$ une valeur constante tres petite.

Si d'abord le point I décrit $\sigma$, le conoïde modifié, ainsi que la région qui lui est intérieure ${ }^{1}$, balaye un volume $T_{, 2 a}$, très peu différent de $T_{2 a}$, et dont la frontière, enveloppe de $\bar{\Gamma}_{12}$, sera constituée de deux parties: l'une $\Gamma_{12}$ très peu différente de $\Gamma_{2}$, lieu des points 2 tels que la trace $\sigma_{-}$, correspondante soit tangente extérieurement à $\sigma$; l'autre $G_{n}$, lieu des points tels que les deux traces dont il vient d'être question soient tangentes entre elles intérieurement, et très peu différente de $G$.

Si au lieu de décrire $\sigma$, le point I décrit toute la multiplicité $\bar{S}_{1}$ dont $\sigma$ est la frontière, le conoïde modifié balaye un volume $T_{12}$ peu différent de $T_{2}$, volume qui est divisé par la cloison interne $G$, en deux parties, distinguées l'une de l'autre par le fait que $\sigma$, est intérieure à $S_{1}$ dans la partie centrale et sécante à $\sigma$ dans la partie annulaire. La frontière de ce volume $T_{12}$ est constituée pour une partie par $I_{\prime_{2}}$ et pour le reste, par le lieu des points 2 tels que le conoïde modifié qu'on en déduit soit tangent à $S_{1}$ (la nappe qui contient le point de contact étant, par ailleurs, extérieure à $T_{2}$ ).

1 Ies volumes balayés par $\dddot{\Gamma}_{, 2}$ et par $\bar{T}_{, 2}$ ne seraient plus identiques entre eux dans la disposition de la figure 2 bis. 
Le principe de Huyghens dans le cas de quatre variables indépendantes. 223

Il est clair qu'un volume $T_{, 2 a}^{\prime}$, tont analogue à $T_{, 2 a}$, serait engendré (avec

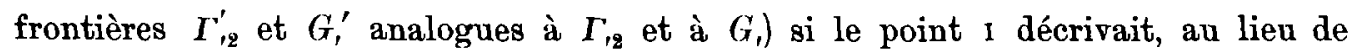
$\sigma$, une frontière modifiée $\sigma^{\prime}$.

Les deux parties $\Gamma_{12}$ et $G_{1}$ de la frontière de $T_{, 2 a}$ découpent respectivement sur $S_{2}$ deux traces, l'une $S_{12}$, voisine de celle du front inteme, l'autre $\sigma_{, 2}$ voisine de $\sigma_{2}$.

III.

9. Ces explications et notations géométriques étant acquises, nous pouvons aborder l'exécution du calcul que nous arons en vue. Nous supposons $u_{0}$ exprimé à l'aide des valeurs $u_{1}, u_{1}^{\prime}$ de $u$ et de sa dérivée transversale $\frac{d u}{d \nu}$ aux divers points de $S_{1}$, en écrivant à cet effet la formule (I) dans laquelle $u, u^{\prime}, L, \nu$ seront affectés de l'indice I et $V, \mathcal{V}$ du double indice or. Dans tout ce qui va suivre, il est entendu que le sens de la normale à $S_{1}$ et, par conséquent $(Y$, p. 63), celui de la transversale $v$ seront ceux qui sont dirigés vers l'intérieur de $T_{1}$. La formule comprendra les sept termes (2). A la rigueur, d'ailleurs, nous pourrions traiter de l'équation sans second membre et, par conséquent, faire abstraction des termes $(a)$ et $(d)$. Mais on va voir que l'introduction de ces termes n'offre que des avantages.

La quantité $u_{1}$ qui figure dans la première formule ainsi écrite peut à son tour (si on la multiplie par $2 \pi$ ) s'exprimer en fonction des données de Cauchy $u_{2}, u_{2}^{\prime}$ prises sur $S_{2}$ par une formule toute semblable, mais où $u, u^{\prime}, L, v$ (transversale dirigée vers $T_{2}$ ) seront affectés de l'indice $2 ; V, \mathcal{V}$, du double indice I 2 . Il en résultera pour $u_{1}^{\prime}$, par différentiation, une expression contenant également $\varphi_{2}, u_{2}, u_{2}^{\prime}$. Reportant dans la valeur de $u_{0}$, nous aurons une expression que nous nous proposerons d'ordonner par rapport aux valeurs de $\varphi_{2}, u_{2}, u_{2}^{\prime}$. Le coefficient d'une valeur quelconque de $\varphi_{z} d T_{2}$, sous un signe $\iiint \int$, sera la valeur correspondante de $-2 \pi \mathcal{V}_{02}$, de même que le coefficient de $u_{2}^{\prime} d S_{z}$ sous un signe $\iiint$. Le coefficient de $\varphi_{2} \frac{d T_{9}}{d \gamma_{2}}$, sous un signe $\iiint$, ou le coefficient de $u_{\mathrm{a}}{ }^{\prime} \frac{d S_{z}}{d \gamma_{z}}$ sous un signe $\iint_{\sigma}$, sera la valeur correspondante de $2 \pi V_{02}$. Enfin, les 
coefficients de $u_{z} d S_{2}, u_{2} \frac{d S_{2}}{d \gamma_{2}}$ sous les signes $\iiint_{S}$ ou $\iint_{\sigma}$ devront être liée aux fonctions $\mathcal{V}_{02}$ et $V_{02}$ par des relations analogues à celles qui résultent de la forme des termes $(b),(f),(g)$. Quant aux termes en $\frac{d T_{2}}{d \gamma_{z}}$ ou $\frac{d S_{2}}{d \gamma_{z}}$ relatifs au front interne, ils devront disparaître.

L'expression que nous avons à former sera évidemment une somme de termes $(a),(d),(b a),(b b),(b c)$ obtenus en combinant chaque terme de la première formule sauf $(a)$ et $(d)$, avec chaque terme de la seconde, absolument (à la commutativité près) comme on le fait dans la multiplication de deux polynômes. Si d'ailleurs on a calculé tous les termes dont le second facteur est $(a)$, on est dispensé de recommencer avec $(c)$, car il suffit de remplacer $\varphi_{2} d T_{2}$ par $u_{2}^{\prime} d \dot{S}_{2}$ avec changement correspondant de l'intégration; et aussi avec $(b)$, le changement étant alors celui de $\varphi_{2} d T_{2}$ en $u_{2} d S_{2}$, mais combiné, cette fois, avec le remplacement de $\mathcal{V}_{12}$ par l'expression $\mathcal{W}_{12}=\frac{d \mathcal{V}_{12}}{d \nu_{2}}-L_{2} \mathcal{V}_{12}$

De même, le calcul d'un terme ayant comme second facteur $(d)$ dispensera, pour des raisons analogues, des termes correspondants à second facteur $(e)$ ou $(f)$. Les termes en $(g)$ (comme second facteur) exigeraient un calcul nouveau; mais on peut s'en dispenser quant au présent, sauf à titre de vérification, en remarquant que les coefficients de $u_{2}$ se déduisent de ceux de $u_{2}^{\prime}$ ainsi qu'il a été expliqué tout à l'heure.

En résumé, on voit qu'on peut se borner à prendre les termes $(b),(c),(e)$, $(f),(g)$ au premier facteur; les termes $(a),(d)$ au second: soit en tout dix expressions à calculer.

Les termes $(e),(f),(g)$ qui figurent comme premiers facteurs seront pris sous leur première forme (2). Au contraire, il y aura lieu de prendre sous la forme $\left(2^{\prime}\right)$ le terme $(d)$ qui figure en second facteur et, de même (toujours en second facteur) $(e),(f)$ ou $(g)$.

Les figures schématiques jointes aux calculs sont de deux espèces. Les figures portant l'indice I $\left(b a_{1}, b d_{1}\right.$, etc. $)$ sont relatives à l'intégration sous sa première forme (intégration extérieure par rapport au point $I$, intérieure par rapport à 2): elles représentent des constructions effectuées dans l'espace $T$ à quatre dimensions et, par conséquent le schéma fait abstraction de deux dimensions. Les figures $b a_{2}, b d_{2}$, etc., qui se rapportent à la forme finale de chaque terme - intégration extérieure par rapport à 2 , intérieure par rapport à I - 
Le principe de Huyghens dans le cas de quatre variables indépendantes. 225 schématisent cette intégration intérieure: elles sont censées tracées sur la multiplicité trois fois étendue $S_{1}$ et, pour cela, il y est fait partout abstraction d'une dimension. ${ }^{1}$

Ainsi, la surface sphéroïdale $\sigma$ est représentée par deux points sur les figures $b a_{1}, b d_{1}$, etc., et par une ligne sur les figures $b a_{2}, b d_{2} \ldots$

10. Terme $(b a)$. - En posant $\frac{d \mathcal{V}_{01}}{d \nu_{1}}-L_{1} \mathcal{V}_{01}=\mathcal{W}_{01}$, il vient

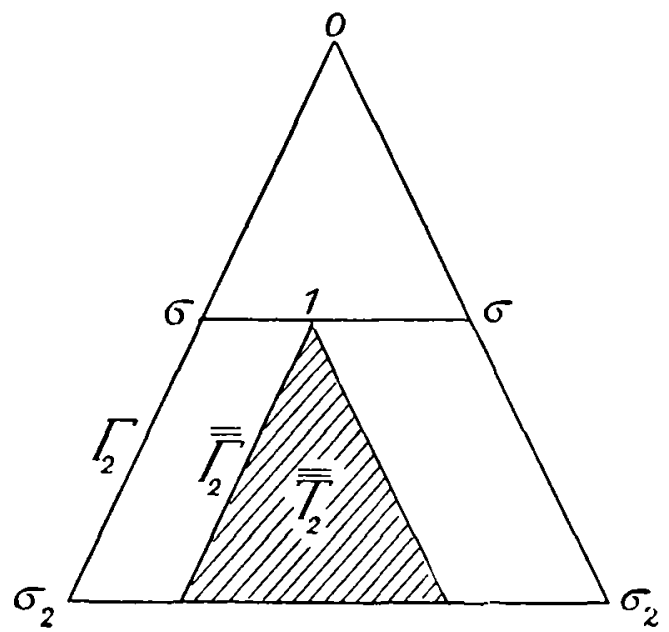

Fig. $(b a)_{1}$

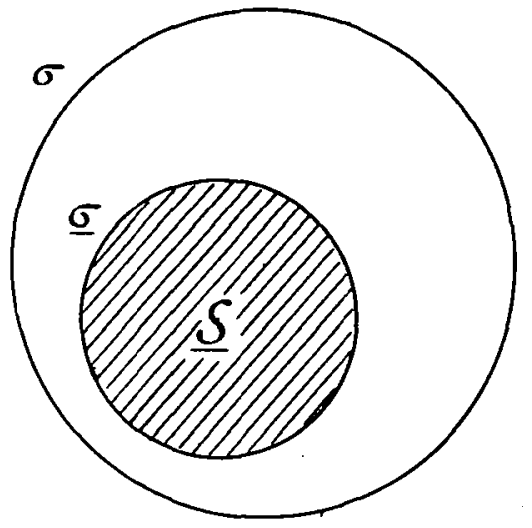

$\left(T_{2} c\right)$

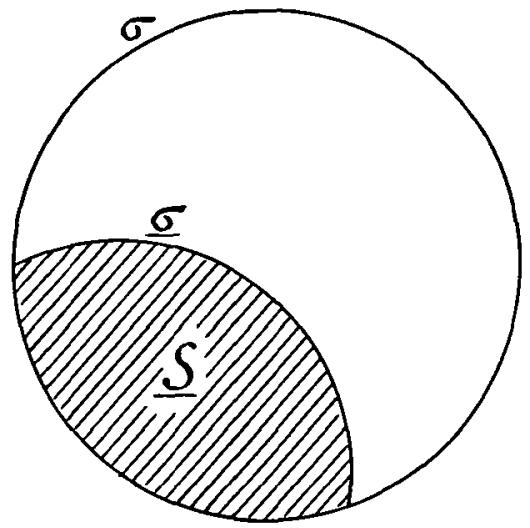

$\left(T_{2} a\right)$

Fig. $(b a)_{2}$

1 Les figures d'indice 2 ponrront d'ailleurs avoir plusieurs aspects, suivant la situation du point 2. En figurant ces aspects, nous indiquerons, par une parenthèse placée sous la figure, les régions de $T_{2}$ auxquelles ils se rapportent.

29-2661. Acta mathematica. 49. Imprimé le 6 juillet 1926. 


$$
(b a)=-\iiint_{S_{1}} \mathcal{W}_{01} d S_{1}\left(\iiint \int_{\bar{T}_{2}} \mathcal{V}_{1 \&} \varphi_{2} d T_{2}\right)
$$

l'intégration extérieure, étant étendue à $S_{1}$ l'intégration intérieure, pour chaque position du point $\mathrm{I}$, au volume $\bar{T}_{2}$ correspondant. Le tout donne une intégration septuple par rapport aux coordonnées ordinaires ou curvilignes de deux points variables l'un sur $S_{1}$, l'autre dans $T_{2}$ et assujettis, l'un par rapport à l'autre, à l'inégalité $\Gamma_{12} \geq 0$. Il est clair que le même résultat peut s'écrire en intervertissant l'ordre des intégrations, soit

$$
(b a)=-\iiint \int_{T_{2}} \varphi_{2} d T_{2}\left(\iiint_{S_{1}} \mathcal{W}_{01} \mathcal{V}_{12} d S_{1}\right)
$$

Conformément à la remarque faite ci-dessus, les termes $(b b),(b c)$ donneraient des résultats tout analogues. Dans $(b c)$, on aurait la même intégrale intérieure avec remplacement, dans l'intégration extérieure, de $\varphi_{2} d T_{2}$ par $u_{2}^{\prime} d S_{2}$, soit

$$
(b c)=-\iiint u_{2}^{\prime} d S_{2}\left(\iiint \mathcal{W}_{01} \mathcal{V}_{12} d S_{1}\right)
$$

dans $(b b)$, il faudrait, en outre, changer $\mathcal{V}_{12}$ en la quantité $\mathcal{W}_{12}=\frac{d \mathcal{V}_{12}}{d \nu_{2}}-L_{2} \mathcal{V}_{12}$ analogue à $\mathcal{W}_{01}$, soit

$$
(b b)=\iiint_{S_{2}} u_{2} d S_{2}\left(\iiint_{\underline{S}_{1}} \mathcal{W}_{01} \mathcal{W}_{12} d S_{1}\right) .
$$

Dans les deux cas, l'intégration extérieure est étendue à toute l'aire $S_{2}$, l'aire $S_{1}$ étant, par contre, d'après ce que nous avons vu, de forme différente (voir la figure) suivant que le point 2 appartient à la région centrale $S_{2 c}$ ou à la région annulaire $S_{2 a}$. Une remarque analogue s'applique d'ailleurs à notre première formule relative à $(b a)$.

11. Terme $(b d)$. - Nous emploierons la seconde forme du terme $(d)$, qui donne 


$$
\begin{aligned}
(b d)=-\iiint_{S_{1}} \mathcal{W}_{01} d S_{1} \frac{d}{d \gamma_{2}} \iiint \int_{\bar{T}_{T_{2}}} V_{12} \varphi_{2} d \Gamma_{2}= \\
=-\frac{d}{d \gamma_{2}}\left[\iiint_{S_{1}} \mathcal{W}_{01} d S_{1} \iiint \int_{\overline{\bar{T}}_{1_{2}}} V_{12} \varphi_{2} d \Gamma_{2}\right] .
\end{aligned}
$$

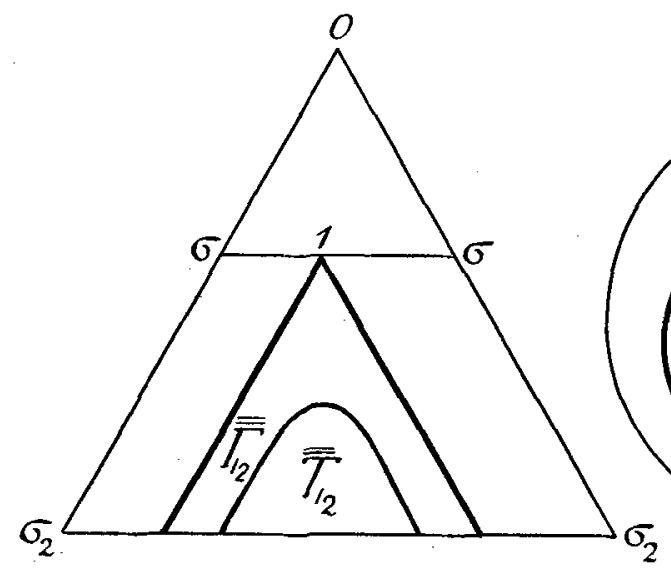

Fig. $(b d)_{1}$

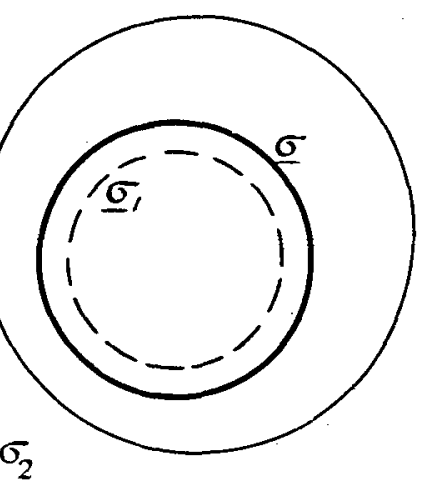

$(T, 2 c)$

Fig. $(b d)_{2}$

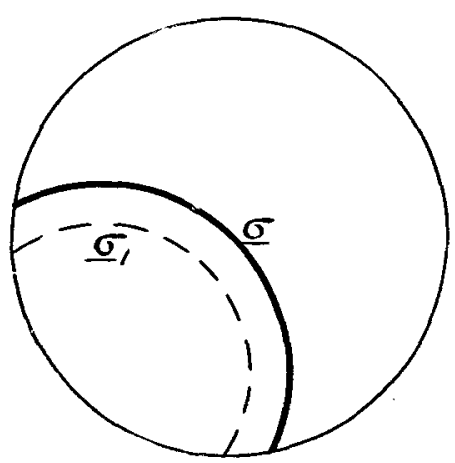

$(T, 2 a)$

Dans la quantité entre crochets, l'interversion des intégrations est possible comme tout à l'heure, les deux points I, 2 variant séparément dans $S_{1}$ et dans $T_{2}$ en étant assujettis à l'inégalité $\Gamma_{12} \geq \gamma_{2}$ : on a ainsi

$$
(b d)=-\frac{d}{d \gamma_{2}}\left[\iiint \int_{T_{, 2}} \varphi_{2} d T_{2}\left(\iiint_{\underline{S}_{1}} \mathcal{W}_{01} V_{12} d S_{1}\right)\right]
$$

L’intégrale extérieure, fonction de la position du point 2 , représente une fonction différente suivant qu'on est dans la région centrale ou dans la région annulaire. Il est clair, cependant, qu'elle reste continue au passage du front interne; d'autre part, elle s'annule sur la frontière $\Gamma_{12}$. Donc il suffit de différentier sous le signe intégral extérieur et l'on a

$$
(b d)=-\iiint \int_{T_{2}} \varphi_{2} d T_{2} \frac{d}{d \gamma_{2}} \iiint_{\underline{\underline{\prime}}} \mathcal{W}_{0 \mathrm{1}} V_{12} d S_{1}=\iiint \int_{T_{2}} \varphi_{2} d T_{2} \iint_{\underline{\sigma}} \mathcal{W}_{0 \mathrm{t}} V_{12} \frac{d S_{1}}{d \gamma_{2}}
$$


Tout pareillement, on aura

$$
(b f)=\iiint_{S_{2}} u_{2}^{\prime} d S_{2} \iint_{\sigma} \mathcal{W}_{01} V_{12} \frac{d S_{1}}{d \gamma_{2}}
$$

Le terme (be) introduira la quantité

$$
W_{12}=\frac{d V_{12}}{d \nu_{2}}-L_{2} V_{19}-\frac{d \Gamma_{12}}{d \nu_{2}} \mathcal{V}_{12}
$$

on aura

$$
(b e)=-\iiint_{S_{2}} u_{2} d S_{2} \iint_{\underline{\sigma}} \mathcal{W}_{01} W_{12} \frac{d S_{1}}{d \gamma_{2}}
$$

12. Terme $(c a)^{1}$ :

$$
(c a)=+\iiint_{S_{1}} \mathcal{V}_{01} d S_{1} \frac{d}{d \nu_{1}} \iiint \int_{\overline{\bar{T}}_{2}} \mathcal{V}_{12} \varphi_{2} d T_{2}
$$

La différentiation de l'intégrale intérieure introduira, outre un terme différentié sous $\iiint \int$

$$
\iiint \int \frac{d \mathcal{V}_{12}}{d \nu_{1}} \varphi_{2} d T_{2}
$$

lequel se traite comme $(b a)$, un terme de frontière. Ce dernier se calculera par la règle énoncée plus haut. L'équation $\Gamma_{12}=0$, qui définit la frontière de $\overline{\bar{T}}_{2}$, dépend du paramètre $\nu_{1}$ si le point I se déplace sur la transversale à $S_{1}$. Le terme de frontière sera donc

$$
\iiint_{\overline{\bar{\Gamma}}_{2}} \mathcal{V}_{12} \varphi_{2} \frac{d \Gamma_{12}}{d \nu_{1}} \cdot \frac{d T_{2}}{d \gamma_{2}}
$$

sans inscription du signe - provenant de la formule (6), parce que, d'autre part, dans le voisinage de $\overline{\bar{\Gamma}}_{2}$, le côté extérieur au domaine d'intégration $\overline{\bar{T}}_{2}$ est celui

${ }^{1}$ Ce terme ne nécessite pas de figure spéciale, les intégrations auxquelles il conduit se traitant soit comme celles qu'introduit $(b a)$, soit comme celles qu'introduit $(b d)$. 
Le principe de Huyghens dans le cas de quatre variables indépendantes.

229

qui correspond à $d \Gamma_{12}<0$. Ceci peut encore s'écrire

$$
-\frac{d}{d \gamma_{2}} \iiint \int_{\bar{T}_{, 2}} \mathcal{V}_{12} \varphi_{2} \frac{d \Gamma_{12}}{d \nu_{1}} d T_{2}
$$

moyennant quoi, le terme résultant de l'intégration extérieure pourra se traiter comme $(b d)$, donnant

$$
\begin{aligned}
-\frac{d}{d \gamma_{2}} \iiint \int_{T_{12}} \varphi_{2} d T_{2} \iiint_{S_{1}} \mathcal{V}_{01} \mathcal{V}_{12} \frac{d \Gamma_{12}}{d v_{1}} d S & = \\
& =-\iiint \int_{T_{2}} \varphi_{2} d T_{2} \frac{d}{d \gamma_{2}} \iiint_{\underline{S}_{1}} \mathcal{V}_{01} \mathcal{V}_{12} \frac{d \Gamma_{12}}{d \nu_{1}} d S
\end{aligned}
$$

puisque, ici encore, l'intégrale intérieure du premier membre est continue et s'annule sur $\Gamma_{12}$.

Done au total,

$$
(c a)=\iiint \int_{T_{2}} \varphi_{2} d T_{2}\left[\iiint_{\underline{S}} \mathcal{V}_{01} \frac{d \mathcal{V}_{12}}{d \nu_{1}} d S_{1}+\iint_{\underline{G}} \mathcal{V}_{01} \mathcal{V}_{12} \frac{d \Gamma_{12}}{d \nu_{1}} \frac{d S_{1}}{d \gamma_{2}}\right]
$$

De même

$$
(c c)=\iiint_{S_{2}} u_{2}^{\prime} d S_{2}\left[\iiint_{\underline{S}} \mathcal{V}_{01} \frac{d \mathcal{V}_{12}}{d \nu_{1}} d S_{1}+\iint_{\underline{\sigma}} \mathcal{V}_{01} \mathcal{V}_{12} \frac{d \Gamma_{12}}{d \nu_{1}} \frac{d S_{1}}{d \gamma_{2}}\right]
$$

pendant que

$$
(c b)=-\iiint_{S_{2}} u_{2} d S_{2}\left[\iiint_{\underline{S}} \mathcal{V}_{01} \frac{d \mathcal{W}_{12}}{d \nu_{1}} d S_{1}+\iint_{\underline{\sigma}} \mathcal{V}_{01} \mathcal{W}_{12} \frac{d \Gamma_{12}}{d \nu_{1}} \frac{d S_{1}}{d \gamma_{2}}\right]
$$

\section{Terme $(c d)^{1}$}

1 Les constructions relatives au calcul de $(c d)$ ne diffèrent de celles qui se rapportent au calcul de $(b d)$ que par la présence, dans le résultat final, d'un terme intégral étendu d̀ la trace modifiée $\sigma$, et différentié par rapport à $\gamma_{2}$. La ligne représentative de $\sigma$, a été tracée d'un trait spécial (trait mixte foreé) sur les figures $(c d)_{2}$, afin de rappeler cette circonstance. 


$$
\begin{aligned}
(c d)=-\iiint_{S_{1}} \mathcal{V}_{01} d S_{1} \frac{d}{d \nu_{1}} \iiint_{\overline{\bar{T}}_{12}} V_{12} \varphi_{2} \frac{d T_{2}}{d \gamma_{2}} & = \\
& =+\iiint_{S_{1}} \mathcal{V}_{01} d S_{1} \frac{d^{2}}{d \nu_{1} d \gamma_{2}} \iiint \int_{\bar{T}_{, 2}} V_{12} \varphi_{2} d T_{2} .
\end{aligned}
$$

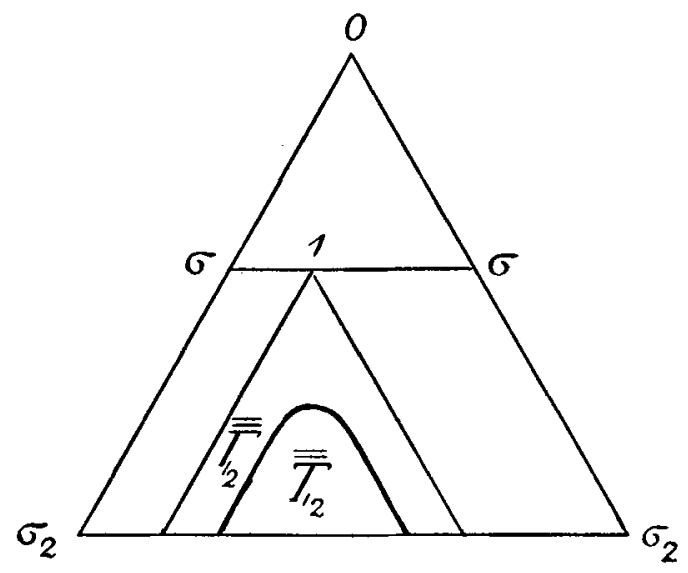

Fig. $(c d)_{1}$

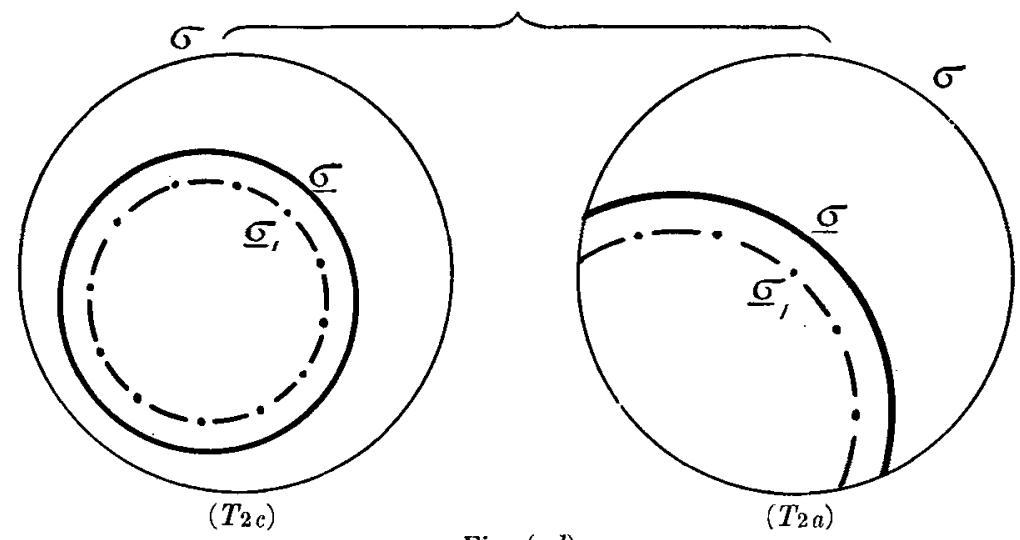

Fig. $(c d)_{2}$

Les variables $\nu_{1}, \gamma_{2}$ étant indépendantes, les différentiations $\frac{d}{d \nu_{1}}, \frac{d}{d \gamma_{2}}$ sont échangeables comme à l'ordinaire; la seconde d'entre elles peut sortir du signe $\iiint$ extérieur, après quoi la première peut se traiter comme tout à l'heure. Ainsi 
Le principe de Huyghens dans le cas de quatre variables indépendantes.

$$
(c d)=\frac{d}{d \gamma_{2}} \iiint \int_{T_{t 2}} \varphi_{2} d T_{2}\left[\iiint_{\underline{S},} \mathcal{V}_{01} \frac{d V_{12}}{d \nu_{1}} d S_{1}+\iint_{\sigma_{g}} \mathcal{V}_{01} V_{12} \frac{d \Gamma_{12}}{d \nu_{1}} \frac{d S_{1}}{d \gamma_{2}}\right]
$$

Comme précédemment (calcul de $(b d)$ ), les deux intégrales entre crochets n'existent que là où $\sigma$, n'est pas entièrement extérieure à $S_{1}$, c'est à dire dans $T_{12}$ et les domaines d'intégration correspondante changent de définition au passage de $G_{,}$, mais sans que ces circonstances donnent lieu à aucun terme spécial, parce que la quantité $\iint$ s'annule sur toute la frontière variable de $T_{12}$ et est continue au passage de la cloison interne. On n'a donc qu'à différentier sous le signe $\iiint \int$ par rapport à $\gamma_{2}$, soit

$$
\begin{array}{r}
(c d)=\iiint \int_{T_{2}} \varphi_{2} d T_{2} \cdot\left[\frac{d}{d \gamma_{2}} \iiint_{\underline{S}_{,}} \mathcal{V}_{01} \frac{d V_{12}}{d \nu_{1}} d S_{1}+\frac{d}{d \gamma_{2}} \iint_{\sigma_{\sigma_{1}}} \mathcal{V}_{01} V_{12} \frac{d \Gamma_{12}}{d \nu_{1}} \frac{d S_{1}}{d \gamma_{2}}\right] \\
=\iiint \int_{T_{2}} \varphi_{2} d T_{2}\left(-\iint_{\sigma} \mathcal{V}_{01} \frac{d V_{12}}{d \nu_{1}} \frac{d S_{1}}{d \gamma_{2}}+\frac{d}{d \gamma_{2}} \iint_{\sigma_{r}} \mathcal{V}_{01} V_{12} \frac{d \Gamma_{12}}{d \nu_{1}} \frac{d S_{1}}{d \gamma_{2}}\right)
\end{array}
$$

de même

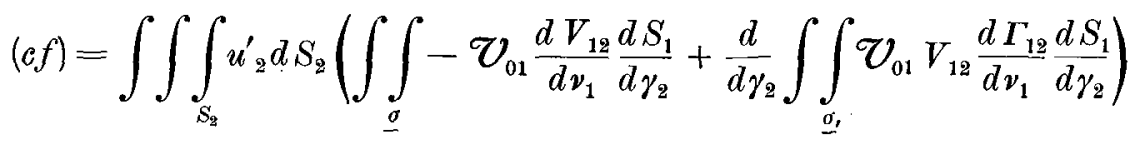

et

$$
(c e)=-\iiint_{S_{2}} u_{\Omega} d S_{\mathrm{Q}}\left(\iint_{\underline{\sigma}}-\mathcal{V}_{01} \frac{d W_{12}}{d \nu_{1}} \frac{d S_{1}}{d \gamma_{2}}+\iint_{\underline{\sigma_{9}}} \mathcal{V}_{01} W_{12} \frac{d \Gamma_{12}}{d \nu_{1}} \frac{d S_{1}}{d \gamma_{2}}\right)
$$

14. Terme (ea). En posant encore $W_{01}=\frac{d V_{01}}{d \nu_{1}}-L_{1} V_{01}-\mathcal{V}_{01} \frac{d \Gamma_{01}}{d \nu_{1}}$, on a

$$
(e a)=\iint_{\sigma} W_{01} \frac{d S_{1}}{d \gamma_{1}} \cdot \iiint \int_{\frac{\bar{T}_{2}}{T_{2}}} \mathcal{V}_{12} \varphi_{2} d T_{2}
$$

ou

$$
(e a)=\iiint \int_{T_{2}} \varphi_{2} d T_{2} \iint_{\bar{\sigma}} W_{01} \mathcal{V}_{12} \frac{d S_{1}}{d \gamma_{1}}
$$




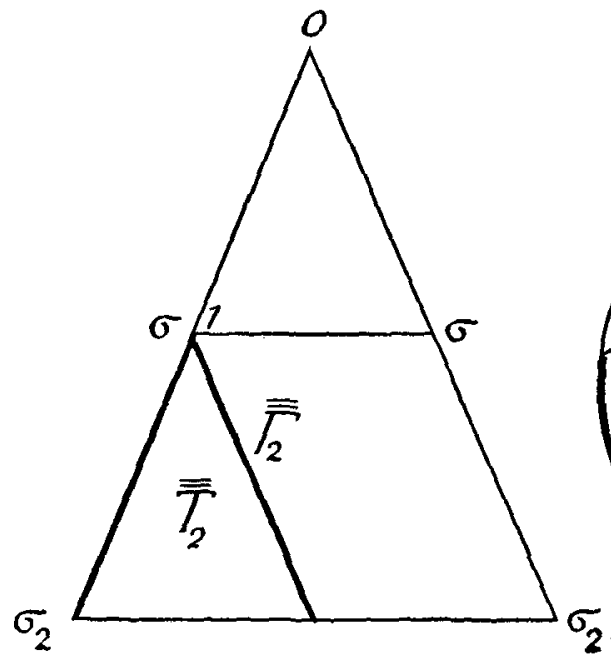

Fig. $(e a)_{1}$

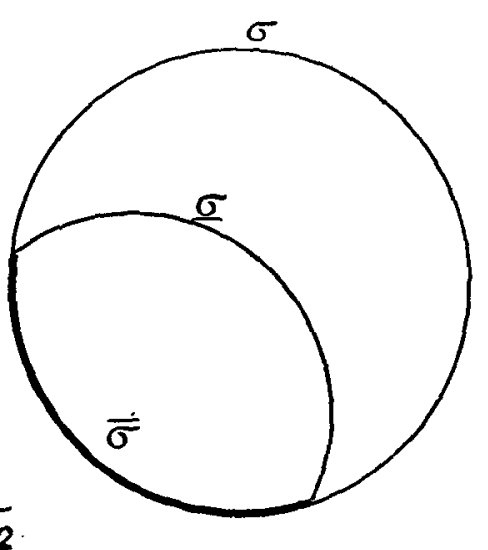

Fig. $(e a)_{2}$

l'intégrale intérieure n'étant relative qu'à la calotte de $\bar{\sigma}$ intérieure à $S_{1}$ (laquelle n'existe que si le point 2 est dans la région annulaire $T_{2 a}$ ).

De même, on aurait

$$
(e c)=\iiint_{S_{2}} u_{2}^{\prime} d S_{2} \iint_{\sigma} W_{01} \mathcal{V}_{12} \frac{d S_{1}}{d \gamma_{1}}, \quad(e b)=-\iiint_{S_{2}} u_{2} d S_{2} \iint_{\bar{\sigma}} W_{01} \mathcal{W}_{12} \frac{d S_{1}}{d \gamma_{1}},
$$

formules analogues à celles que nous avons obtenues pour $(b a),(b c),(b b)$, ̀̀ ceci près que l'intégration intérieure s'étend à une portion de $\sigma$ et non à une portion de $S_{1}$.

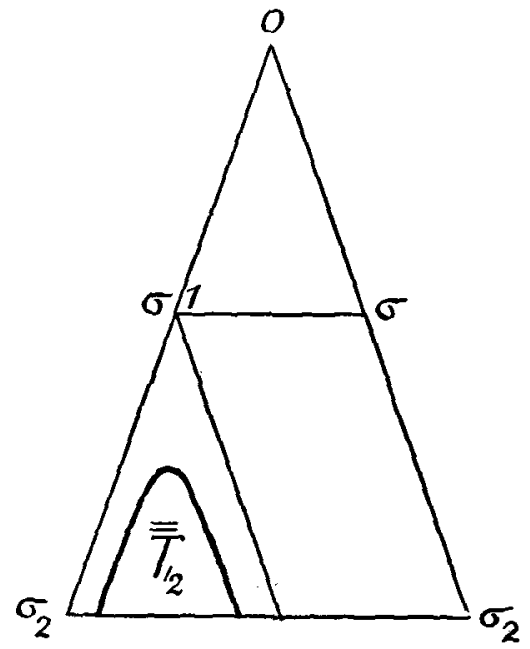

Fig. $(e d)_{1}$

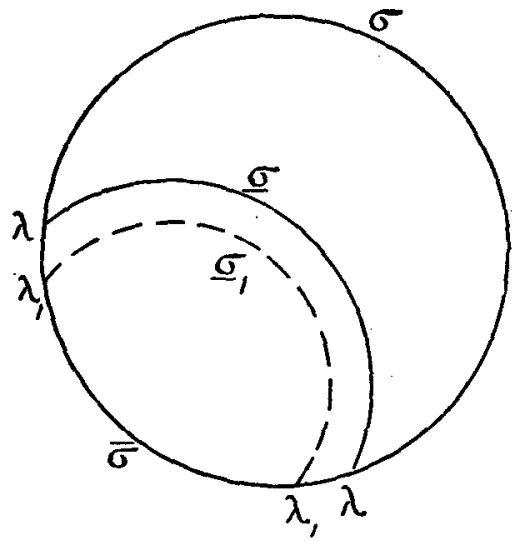

Fig. $(e d)_{2}$ 
Le principe de Huyghens dans le cas de quatre variables indépendantes. 233

Terme $(e d)$. - La valeur du terme $(e d)$ sera dans une relation tout analogue arec celle de $(b d)$ : on aura d'abord

$$
\begin{aligned}
(e d) & =\iint_{\sigma} W_{01} \frac{d S_{1}}{d \gamma_{1}} d \gamma_{2} \iiint \int_{\bar{T}_{12}} V_{12} \varphi_{2} d T_{2} \\
& =\frac{d}{d \gamma_{2}}\left(\iiint \int_{T_{, 2 a}} \varphi_{2} d T_{2} \iint_{\sigma,} W_{01} V_{12} \frac{d S_{1}}{d \gamma_{1}}\right) .
\end{aligned}
$$

$\bar{\sigma}$, est la partie de $\sigma$ située à l'intérieur de $S_{11}$, c'est à dire une calotte ayant pour frontière la ligne $\lambda_{1}$ d'intersection de $\sigma$ avec $\Gamma_{12}=\gamma_{2}$. L'intégrale intérieure s'annulant ̀̀ la frontière du domaine d'intégration extérieure, la différentiation portera exclusivement sur cette intégrale intérieure et se fera à vue par l'emploi

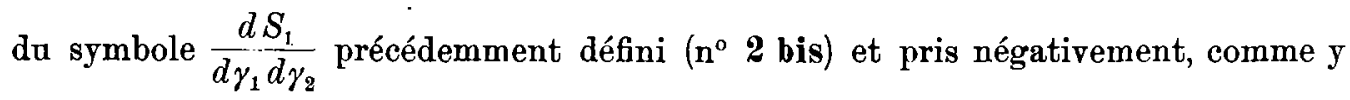
conduit la règle indiquée au $n^{\circ} 2$, lorsqu'on tient compte de la note de la p. 2 Io. Ainsi

$$
(e d)=-\iiint \int_{T_{2}} \varphi_{2} d T_{2} \int_{2} W_{01} V_{12} \frac{d S_{1}}{d \gamma_{1} d \gamma_{2}}
$$

et

$$
(e f)=-\iiint_{S_{2}} u_{2}^{\prime} d S_{2} \int_{\lambda} W_{01} V_{12} \frac{d S_{1}}{d \gamma_{1}} d \gamma_{2}^{-}, \quad(e e)=\iiint_{\Sigma_{2}} u_{2} d S_{2} \int_{\lambda} W_{01} W_{12} \frac{d S_{1}}{d \gamma_{1} d \gamma_{2}}
$$

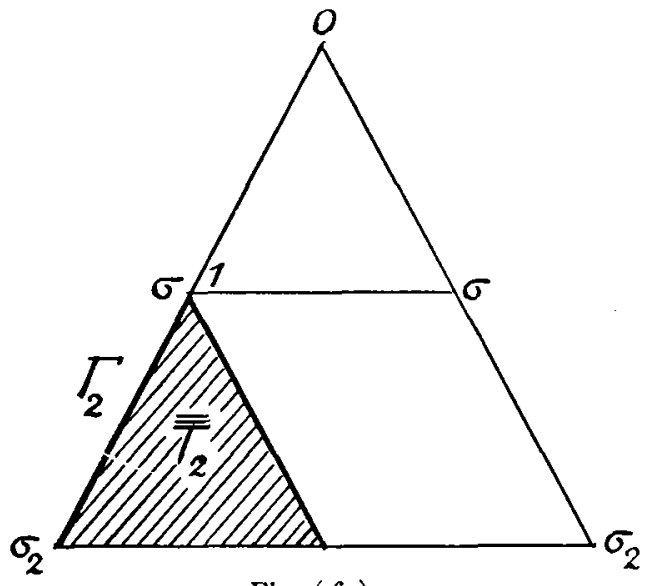

Fig. $(f a)_{1}$

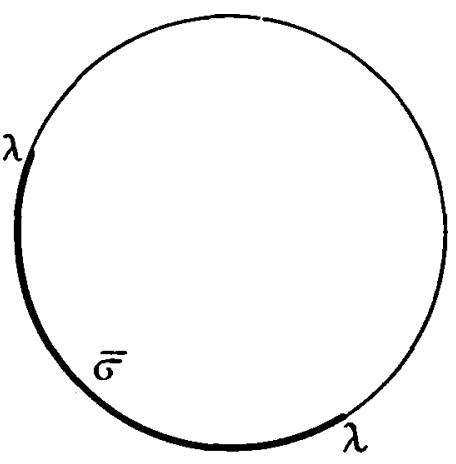

Fig. $(f a)_{2}$

30-2661. Acta mathematica. 49. Imprimé le 6 juillet 1926. 
15. Terme $(f a)$. - Les termes en $(f)$ se traiteront évidemment en principe comme les termes en (c), à des changements près semblables à ceux qui se présentent dans les termes en (e) par rapport aux termes en (b).

$$
(f a)=-\iint_{\sigma} V_{01} \frac{d S_{1}}{d \gamma_{1}} \frac{d}{d \nu_{1}} \iiint \int_{\bar{T}_{2}} \mathcal{V}_{12} \varphi_{2} d T_{2}
$$

donnera

$$
(f a)=-\iiint \int_{T_{2} a} \varphi_{2} d T_{2}\left(\iint_{\sigma} V_{01} \frac{d \mathcal{V}_{12}}{d \nu_{1}} \frac{d S_{1}}{d \gamma_{1}}+\int_{\lambda} V_{01} \mathcal{V}_{12} \frac{d \Gamma_{12}}{d \nu_{1}} \frac{d S_{1}}{d \gamma_{1}} d \gamma_{2}\right)
$$

de même que

$$
\begin{aligned}
& (f c)=-\iiint_{S_{2 a}} u_{2}^{\prime} d S_{2}\left(\iint_{\sigma} V_{01} \frac{d \mathcal{V}_{12}}{d \nu_{1}} \frac{d S_{1}}{d \gamma_{1}}+\int_{\lambda} V_{01} \mathcal{V}_{12} \frac{d \Gamma_{12}}{d \nu_{1}} \frac{d S_{1}}{d \gamma_{1} d \gamma_{2}}\right) \\
& (f b)=\iiint_{S_{2} a} u_{2} d S_{2}\left(\iint_{\frac{\sigma}{\sigma}} V_{01} \frac{d \mathcal{W}_{12}}{d \nu_{1}} \frac{d S_{1}}{d \gamma_{1}}+\int_{\lambda} V_{01} \mathcal{W}_{12} \frac{d \Gamma_{12}}{d \nu_{1}} \frac{d S_{1}}{d \gamma_{1} d \gamma_{2}}\right)
\end{aligned}
$$

16. Terme $(f d)$. Tout dabord, en opérant comme pour $(c d)$,

$$
\begin{aligned}
(f d) & =-\iint_{\sigma} V_{01} \frac{d S_{1}}{d \gamma_{1}} \frac{d^{2}}{d \nu_{1} d \gamma_{2}} \iiint \int_{\overline{\bar{T}}_{, 2}} V_{12} \varphi_{2} d T_{2} \\
& =-\frac{d}{d \gamma_{2}} \iiint \int_{T_{, 2 a}} \varphi_{2} d T_{2}\left(\iint_{\sigma_{1}} V_{01} \frac{d V_{12}}{d \nu_{1}} \frac{d S_{1}}{d \gamma_{1}}+\int_{\lambda_{1}} V_{01} V_{12} \frac{d \Gamma_{12}}{d \nu_{1}} \frac{d S_{1}}{d \gamma_{1} d \gamma_{2}}\right)
\end{aligned}
$$

l'intégration quadruple du dernier membre étant encore restreinte au domaine des points 2 pour lesquels $\bar{\sigma}$, existe, c'est à dire à $T_{, 2 a}$.

La différentiation du premier terme donne sans difficulté (l'intégrale $\iint_{\frac{\sigma}{,}}$

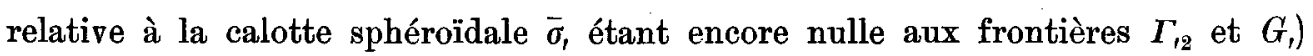

$$
\iiint \int_{T, 2 a} \varphi_{2} d T_{2} \int_{\lambda} V_{01} \frac{d V_{12}}{d \nu_{1}} \frac{d S_{1}}{d \gamma_{1} d \gamma_{2}}
$$




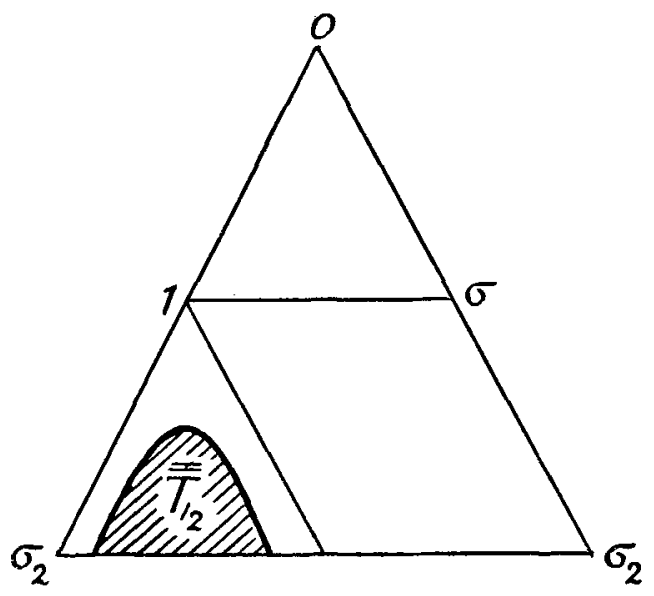

Fig. $(f d)_{1}$

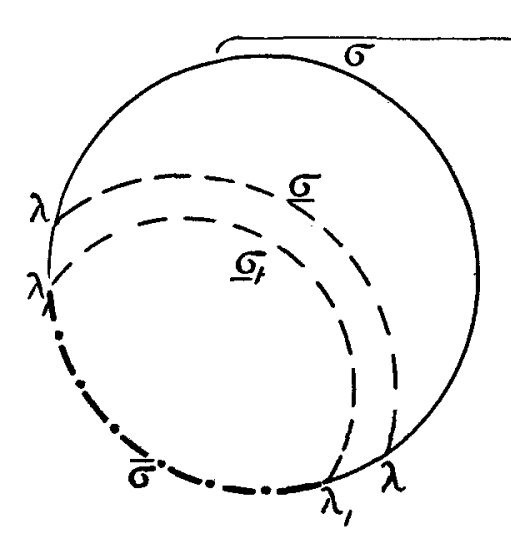

$\left(T_{, 2 a}\right)$

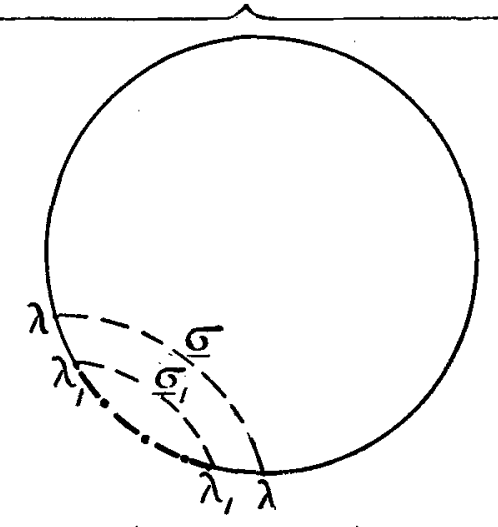

(voisinage de $\Gamma_{, 2}$ )

Fig. $(f d)_{2}$

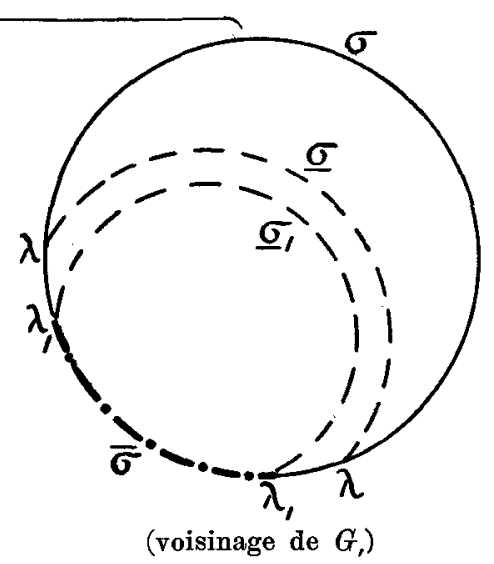

(voisinage de $G$ )

Dans le second terme, l'intégrale suivant $\lambda$, est mise pour

$$
-\frac{d}{d \gamma_{2}} \iint_{\sigma_{1}} V_{01} V_{12} \frac{d \Gamma_{12}}{d v_{1}} \frac{d S_{1}}{d \gamma_{1}}
$$

et il sera nécessaire de lui laisser cette seconde forme pour trouver sa valeur limite aux frontières mentionnées il y a un instant.

S'il est vrai, en effet, que, sur ces frontières, l'aire $\bar{\sigma}$, (ainsi que son contour $\lambda_{1}$ ) et, par conséquent, l'intégrale double correspondante; s'annulent, il n'en est pas de même de la quantité (I I): cette dernière devra alors se calculer non 
par une intégrale simple suivant $\lambda_{t}$, mais par la méthode du no 4 . On devra donc, cette fois, introduire, dans la différentiation de l'intégrale quadruple, deux termes de frontière, où figureront les valeurs limites de l'expression (I I).

Dans le calcul de ces deux termes, la valeur limite de la quantité $V_{01} V_{12} \frac{d \Gamma_{12}}{d \nu_{1}}$ figurera en facteur et peut, dès à présent, sortir du signe $\iint$.

Le facteur restant est la quantité géométrique

$$
q=-\frac{d}{d \gamma_{2}} \iint_{\sigma_{,}} \frac{d S_{1}}{d \gamma_{1}}
$$

dont la signification est, d'après ce qui précède, la suivante. I étant un point pris sur $\sigma ; 2$, un point de $\Gamma_{\mathbf{2}}$ (ou de $G$ ) pris sur la bicaractéristique issue de I ; $\gamma_{2}$, une première constante positive (finalement nulle); $2_{1}$, un point roisin de 2 et situé sur l'enveloppe $\Gamma_{12}$ (ou $G$ ) formée avec cette valeur de $\gamma_{2}$, c'est à dire tel que l'hypersurface $\Gamma_{12}=\gamma_{2}$ correspondante soit tangente à $\sigma ; g_{2}<\gamma_{2}$ un second paramètre infiniment voisin de $\gamma_{2}^{\prime} ; \bar{\sigma}_{t}$, la calotte interceptée par l'hypersurface $I_{18}=g_{2}$ sur $\sigma$, l'expression (I2) est le coefficient de $\gamma_{2}-g_{2}$ dans l'intégrale $\iint_{\bar{\sigma},} \frac{d S_{1}}{d \gamma_{1}}$.

$q V_{01} V_{12} d I_{1 \underline{1}}$ sera la limite de l'intégrale intérieure: elle devra, conformément à la règle précédemment obtenue, être multipliée par le quotient $-\frac{d T_{2}}{d \gamma_{2}}$, quotient (changé de signe ${ }^{1}$ ) par $d \gamma_{2}$ de l'élément de volume compris entre les hypersurfaces voisines $\Gamma_{2}, I_{, 2}$ (ou $G, G$,).

$\Gamma_{12}$ est, par définition, l'enveloppe de l'hypersurface $I_{12}=\gamma_{2}$ lorsque le point I décrit $\sigma$, de sorte que son équation se déduit de $\Gamma_{18}=\gamma_{2}$ en substituant aux coordonnées $d u$ point I leurs valeurs déterminées en fonction de celles du point 2 par la condition que.le premier membre soit stationnaire. C'est en particulier ainsi qu'il faut opérer pour le calcul de la quantité $\frac{d \Gamma_{, 2}}{d n}$ (ou plus précisément $d \boldsymbol{I}_{,}$,
$d n_{2}$

\footnotetext{
${ }^{1}$ Voir le calcul de $(c a)$.
} 
Le principe de Huyghens dans le cas de quatre variables indépendantes. 237 théorie des enveloppes nous apprend que le calcul peut, dans ces conditions, se faire sans tenir compte de la variabilité du point I: elle a la même valeur pour l'enveloppe $\Gamma_{12}$ que, pour l'enveloppée $\bar{I}_{12}$.

Tenant compte des deux termes correctifs ainsi calculés, nous avons

$$
\begin{aligned}
& (f d)=\iiint \int_{T_{2 a}} \varphi_{z} d T_{2}\left(\int_{2} V_{01} \frac{d V_{12}}{d \nu_{1}} \frac{d S_{1}}{d \gamma_{1}} d \gamma_{2}-\frac{d}{d \gamma_{2}} \int_{\lambda_{1}} V_{01} V_{12} \frac{d I_{12}}{d v_{1}} \frac{d S_{1}}{d \gamma_{1}} d \gamma_{2}\right) \\
& -\iiint_{\Gamma_{2}} \varphi_{2} V_{01} V_{12} d \Gamma_{12} q \frac{d T_{2}}{d \nu_{1}}-\iint_{G} \cdots
\end{aligned}
$$

On aura de même

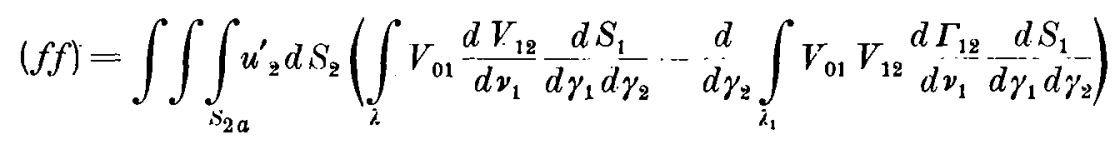

$$
\begin{aligned}
& -\iint_{\sigma_{z}} u_{2}^{\prime} V_{01} V_{12}^{d \Gamma_{12}} d \nu_{1}^{d S_{2}} d \gamma_{z}-\iint_{\beta_{2}}
\end{aligned}
$$

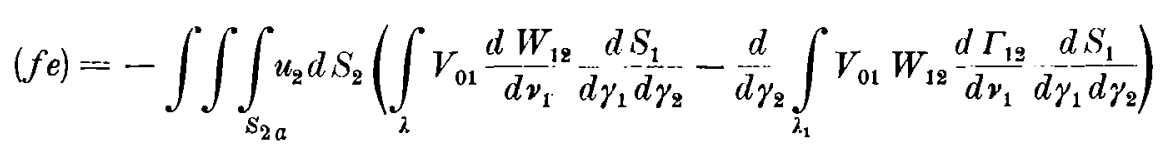

$$
\begin{aligned}
& +\iint_{\sigma_{2}} u_{2} V_{01} W_{12} \frac{d I_{12}}{d \nu_{1}} q \frac{d S_{2}}{d \gamma_{z}}+\iint_{\delta_{3}}
\end{aligned}
$$

17. Occupons nous enfin des termes dans lesquels le premier facteur est $(g)$. Terme $(g a)$. On a

$$
\begin{aligned}
&(g a)=-\frac{d}{d \gamma_{1}} \iint_{\sigma^{\prime}} V_{01} \frac{d \Gamma_{01}}{d \bar{\nu}_{1}} \frac{d S_{1}}{d \gamma_{1}} \iiint \int_{\overline{\bar{T}}_{2}} V_{12} \varphi_{2} d T_{2} \\
&=-\frac{d}{d \gamma_{1}} \iiint \int_{T^{\prime}{ }_{2} a} \varphi_{2} d T_{2} \iint V_{01} V_{12} \frac{d \Gamma_{01}}{d \nu_{1}} d S_{1} \\
& d \gamma_{1}
\end{aligned}
$$

$T_{2 a}^{\prime}$ étant le domaine engendré dans $T_{2}$ à l'aide de $\sigma^{\prime}$ comme $T_{2 a}$ l'est à l'aide de $\sigma$. Ce domaine dépend done de $\gamma_{1}$; mais, comme, sur sa frontière, l'intégrale intérieure s'annule, nous n'aurons pas de terme à écrire de ce chef. Donc 


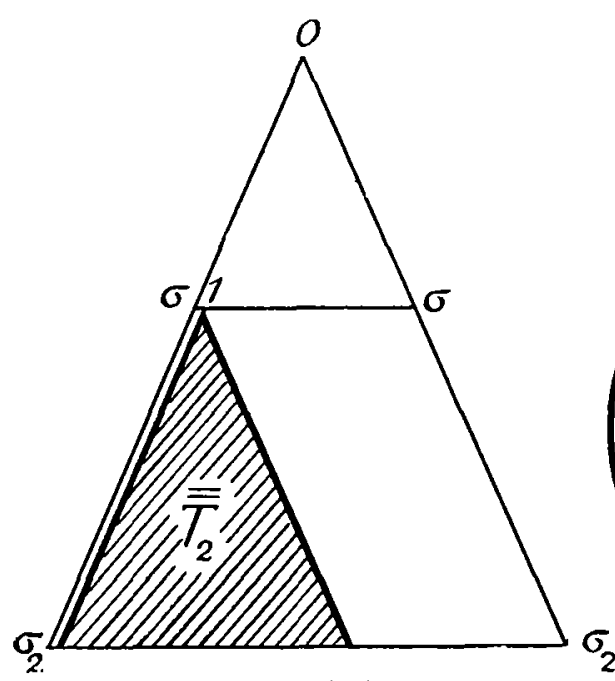

Fig. $(g a)_{1}$

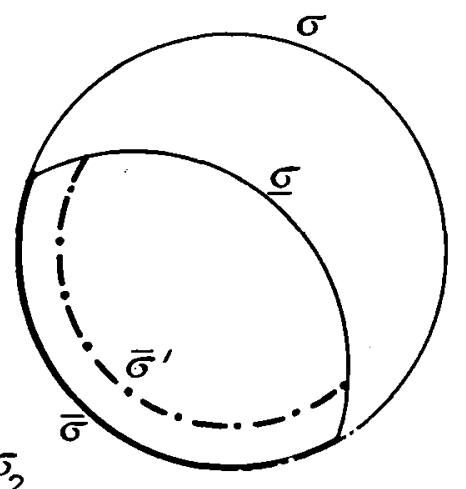

Fig. $(g a)_{2}$

$$
\begin{gathered}
(g a)=-\iiint \int_{T_{2 a}} \varphi_{2} d T_{2} \frac{d}{d \gamma_{1}} \iint_{\sigma^{\prime}} V_{01} V_{12}^{d I_{01}} d S_{1} d \gamma_{1} \\
(g c)=-\iiint_{S_{2 a}} u_{2}^{\prime} d S_{2} \frac{d}{d \gamma_{1}} \iint_{\sigma^{\prime}} V_{01} V_{12} d I_{01} \frac{d S_{1}}{d \nu_{1}} d \gamma_{1} \\
(g b)=\iiint_{S_{2 a}} u_{2} d S_{2} \frac{d}{d \gamma_{1}} \iint_{\sigma^{\prime}} V_{01} W_{12} \frac{d \Gamma_{01}}{d \nu_{1}} \frac{d S_{1}}{d \gamma_{1}}
\end{gathered}
$$

18. Terme $(g d)$.

$$
\begin{aligned}
(g d) & =-\frac{d}{d \gamma_{1}} \iint_{\sigma^{\prime}} V_{01} \frac{d \Gamma_{01}}{d \nu_{1}} \frac{d S_{1}}{d \gamma_{1}} \frac{d}{d \gamma_{9}} \iiint \int_{\bar{T}_{12}} V_{12} \varphi_{2} d T_{2} \\
& =-\frac{d^{2}}{d \gamma_{1} d \gamma_{2}} \iiint \int_{T_{, 2 a}^{\prime}} \varphi_{2} d T_{2} \iint V_{01} V_{12} \frac{d I_{01}}{d \nu_{1}} \frac{d S_{1}}{d \gamma_{1}}
\end{aligned}
$$

les deux différentiations étant échangeables puisque $\gamma_{1}$ et $\gamma_{2}$ sont deux variables indépendantes. Le domaine $T_{, 2 a}^{\prime}$ est celui qui est balayé par $\overline{\bar{T}}_{12}$ lorsque le point I décrit $\sigma^{\prime}$ : il a par conséquent encore une frontière externe $\Gamma_{, 2}^{\prime}$, lieu des points

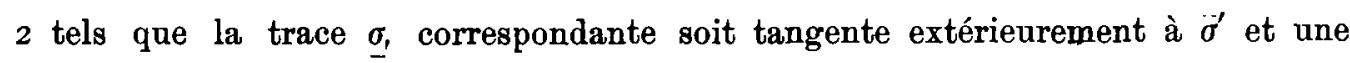


Le principe de Huyghens dans le cas de quatre variables indépendantes. 239

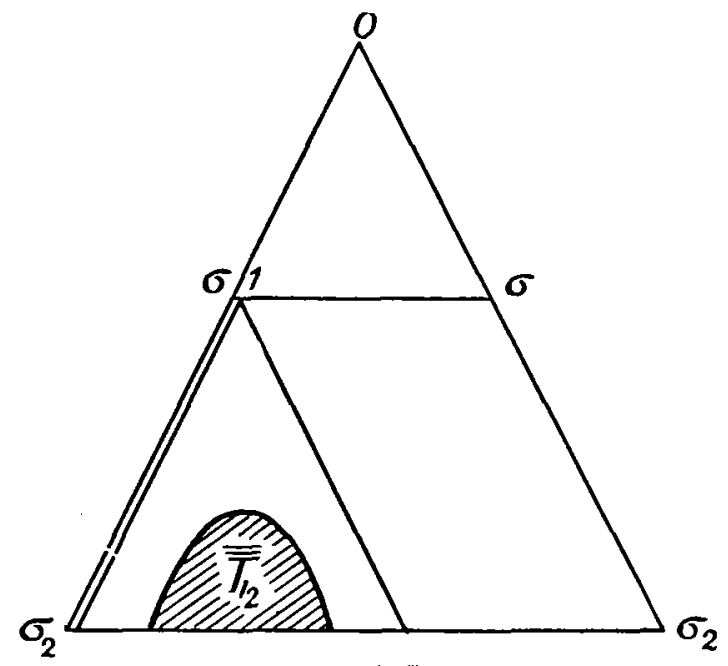

Fig. $(g d)_{1}$

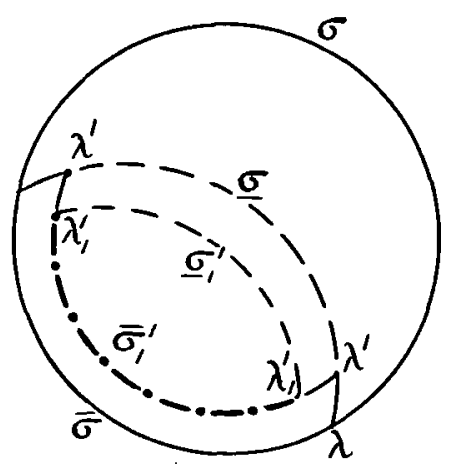

$\left(T_{, 2 a}^{\prime}\right)$

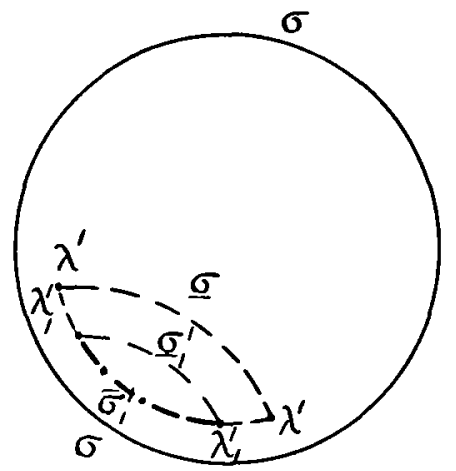

(voisinage de $\Gamma_{,}^{\prime}$ )

Fig. $(g d)_{2}$

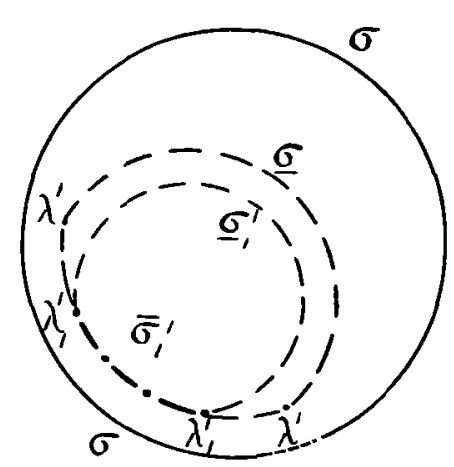

(voisinage de $G$, )

frontière interne $G_{,}^{\prime}$, lieu des points tels que $\sigma_{-}$, soit tangente à $\bar{\sigma}^{\prime}$ intérieurement. Lorsque le point 2 est sur l'une de ces deux frontières, l'aire $\bar{\sigma}$, se réduit à zéro: on peut donc encore se contenter de différentier sous le signe $\iiint \int$ par rapport à $\gamma_{2}$, soit

$$
(g d)=-\frac{d}{d \gamma_{1}} \iiint \int_{T^{\prime} 2 a} \varphi_{2} d T_{2} \frac{d}{d \gamma_{2}} \iint_{\sigma^{\prime}} V_{01} V_{12} \frac{d \Gamma_{01}}{d \nu_{1}} \frac{d S_{1}}{d \gamma_{1}}
$$

ce qu'on peut encore écrire 


$$
(g d)=\frac{d}{d \gamma_{1}} \iiint \int_{T^{\prime} 2_{a}} \varphi_{2} d T_{2} \int_{\lambda^{\prime}} V_{01} V_{12} \frac{d \Gamma_{01}}{d \nu_{1}} \frac{d S_{1}}{d \gamma_{1}} d \gamma_{2}
$$

sous réserve de noter que, aux frontières de $T_{2 a}^{\prime}$, donc sur $\Gamma_{2}^{\prime}$ ou $G^{\prime}$, la vàleur que prend l'intégrale $\int_{\lambda^{\prime}}$ doit être calculée à l'aide de la première forme de cette expression.

Cette valeur frontière n'étant pas nulle, elle donnera lieu dans la différentiation, à un terme dont le calcul se fera comme pour $(f d)$. La quantité $q$ est commune aux deux calculs et, par conséquent, la valeur limite de l'intégrale intérieure y est la même, au changement près de $\frac{d \Gamma_{12}}{d \nu_{1}}$ en $\frac{d \Gamma_{01}}{d \nu_{1}}$.

La valeur limite ainsi obtenue devra être multipliée par $\varphi_{2}$ et par un élément différentiel dont le calcul se fera d'après les principes déjà invoqués dans les cas précédents. $\quad \boldsymbol{I}_{2}^{\prime}$ est l'enveloppe d'un conoïde caractéristique dont le sommet I décrit l'intersection $\sigma^{\prime}$ de $S_{1}$ avec $\Gamma_{01}=\gamma_{1}$. Son équation

$$
\Gamma_{2}^{\prime}\left(x_{1}, \ldots, x_{m}, \gamma_{1}\right)=\mathrm{o}
$$

s'obtiendra donc en éliminant les $m$ - I coordonnées curvilignes du point I entre les équations

$$
\begin{array}{ll}
\left(\overline{\bar{\Gamma}}_{12}\right) & \Gamma_{12}=0, \\
\left(\sigma^{\prime}\right) & \Gamma_{01}=\gamma_{1}
\end{array}
$$

et les $(m-2)$ équations qui expriment que le premier membre de $\left(\overline{\bar{\Gamma}}_{12}\right)$ est stationnaire pour un déplacement du point I sur $\sigma^{\prime}$ (le point I ainsi déterminé étant à la limite, pour $\gamma_{1}=0$, le point $S_{1}$ situé sur la ligne de contact de $\Gamma$ avec le conoïde de sommet 2 , c'est à dire avec la bicaractéristique du point 2). Par exemple, on pourra prendre

$$
\Gamma_{2}^{\prime}=\Gamma_{12}
$$

les coordonnées du point I étant remplacées, au second membre, par leurs valeurs calculées en fonction de $x_{1}, \ldots x_{m}$ (coordonnées du point 2 ) dans les conditions 
Le principe de Huyghens dans le cas de quatre variables indépendantes. 241 qui viennent d'être indiquées. D'après la règle $d u n^{\circ} \boldsymbol{2}$, l'élément d'étendue de $\Gamma_{2}^{\prime}$ à introduire est

$$
d \Sigma:\left(-\frac{d \Gamma_{2}^{\prime}}{d n_{2}}: \frac{\partial \Gamma_{2}^{\prime}}{\partial \gamma_{1}}\right)
$$

$\left(n_{2}\right.$, normale à̀ $\Gamma_{2}^{\prime}$, dirigée en dehors de $\left.T_{2 a}^{\prime}\right)$.

Comme plus haut, les dérivées de $\Gamma_{2}^{\prime}$ se calculeront sans avoir à tenir compte de la variabilité du point I, c'est à dire seront égales aux dérivées correspondantes de $\Gamma_{12}$. Le quotient $d \Sigma: \frac{d \Gamma_{2}^{\prime}}{d n_{2}}$ est donc la même quantité $\frac{d \Gamma_{2}}{d \gamma_{2}}$ que nous avions à considérer dans le calcul de $(f d)$. Quant à $\frac{\partial \Gamma_{2}^{\prime}}{\partial \gamma_{1}}=\frac{d \Gamma_{12}}{d \gamma_{1}}$, ce n'est autre chose que le rapport constant ( $\mathrm{n}^{\circ} 2$ bis) entre $d \Gamma_{12}$ et $d \Gamma_{01}$, pour un déplacement quelconque effectué sur $S_{1}$ à partir du point de contact de $\sigma^{\prime}$ et de $\underline{\sigma}_{\underline{\prime}}$ c'est à dire, à la limite, que le rapport ${ }^{1} \frac{d \Gamma_{12}}{d \Gamma_{01}}$ en un point I de $\sigma$. Des considérations toutes semblables s'appliquant le long de $G^{\prime}$ (done, à la limite, de $G$ ), nous obtenons finalement

$$
\begin{aligned}
(g d)= & \iiint \int_{T_{2 a}} \varphi_{2} d \Gamma_{2} \frac{d}{d \gamma_{1}} \int_{\lambda^{\prime}} V_{01} V_{12} \frac{d \Gamma_{01}}{d \nu_{1}} \frac{d S_{1}}{d \gamma_{1} d \gamma_{2}} \\
& -\iiint_{\Gamma_{2}} q \varphi_{2} V_{01} V_{12} \frac{d \Gamma_{01}}{d \nu_{1}} \frac{d T_{2}}{d \gamma_{2}} \frac{d \Gamma_{12}}{d \Gamma_{01}}-\iiint_{G},
\end{aligned}
$$

avec formules correspondantes pour $(g f),(g e)$ (cette dernière toujours avec changement de $V_{12}$ en $W_{12}$ ).

19. L'ensemble des coefficients de $\varphi_{2} d T_{2}$ sous les signes d'intégration quadruple donnera la valeur (changée de signe et multipliée par $2 \pi$ ) de $\mathcal{V}_{02}$; on a ainsi (en remplaçant $\mathcal{W}$ et $W$ par leurs valeurs):

${ }^{1}$ D'après la formation et les propriétés $(Y, 58)$ de la quantité $\Gamma$, ce rapport n'est autre qu' le rapport (négatif) entre les valeurs de la variable $s$ (loc. cit.) qui, comptées a partir du point I correspondent respectivement aux points 2 et 0 . Cette remarque n'est valable que sur $\Gamma_{2}$ et nou $\operatorname{sur} G$.

31-2661. Acta mathomatica. 49. Imprimé le 6 juillet 1926. 


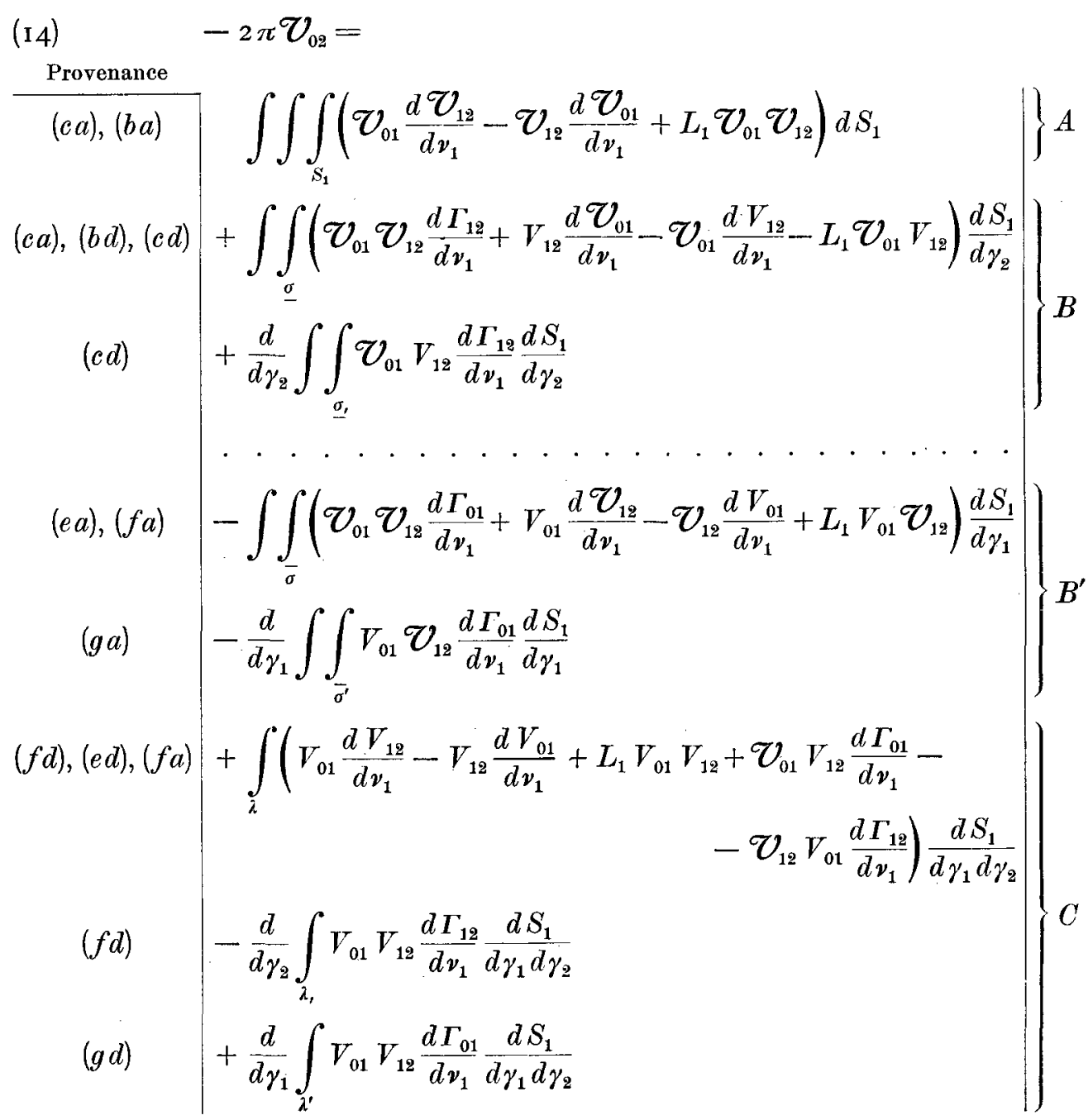

Les termes $B^{\prime}, C$ n'existent que si les deux traces $\sigma, \sigma$ sont sécantes; du moins il en est ainsi dans la disposition de la figure 2 . $\mathrm{Si}$, au contraire, la disposition était celle de la figure 2 bis, ce seraient les termes $B$ qui disparaîtraient dans la partie centrale en même temps que les termes $C$.

La formule manifeste bien, comme cela était nécessaire, une symétrie par rapport aux deux points o et 2 (on sait que le résultat ne doit pas changer par l'échange de ces deux points entre eux, en même temps que de l'équation donnée avec son adjointe).

20. Considérons maintenant ce qui est relatif à $\Gamma_{2}$ et ce qui est relatif au 
Le principe de Huyghens dans le cas de quatre variables indépendantes. 243 front interne. Dans le premier cas, 2 étant un point de $I_{2}$, on doit obtenir la valeur de $2 \pi V_{0 z}$ comme égale au coefficient de $\frac{d T_{2}}{d \gamma}$ sous le signe d'intégration triple. $\gamma$, nous le rappellerons, représente la valeur de $\Gamma_{02}$. Notre formule (termes $(f d),(g d))$ fait intervenir comme élément d'étendue de $\Gamma_{2}$ la quantité $\frac{d T_{2}}{d \gamma_{2}}$, c'est à dire $\frac{d T_{2}}{d I_{12}}$. Mais, en raisonnant comme nous l'avons fait un peu plus haut, on voit que le rapport de ces deux éléments est celui de $d \Gamma_{12}$ à $d \Gamma_{0 z}$ (égal lui-même à celui de valeurs de $s$ correspondant aux arcs 12,02 de bicaractéristique). Nous avons donc

$$
2 \pi V_{02}=\left(\frac{d \Gamma_{02}}{d \Gamma_{12}}\right)_{2}\left[q V_{01} V_{12} \frac{d \Gamma_{1 z}}{d \nu_{1}}+q V_{01} V_{12} d I_{01}\left(\begin{array}{l}
d \nu_{1} \\
d \Gamma_{12}
\end{array}\right)_{1}\right]
$$

Les deux termes ainsi écrits se doublent: car les deux conoïdes $\Gamma_{01}, \Gamma_{12}$ sont tangents au point I et, dès lors, le rapport des dérivées de $\Gamma_{12}$ et de $\Gamma_{01}$, prises suivant la même direction transversale, est égal au rapport constant $\frac{d \Gamma_{12}}{d \Gamma_{01}}$.

21. Au front interne, le coefficient de $\frac{d T_{2}}{d \gamma_{2}}$ est encore la même quantité

$$
q V_{01} V_{12}\left[\frac{d \Gamma_{12}}{d \nu_{1}}+\frac{d \Gamma_{01}}{d \nu_{1}}\left(\begin{array}{l}
d \Gamma_{12} \\
d \Gamma_{01}
\end{array}\right)_{1}\right]
$$

Mais, cette fois, les deux termes entre crochets se détruisent. Ici, en effet, le rapport $\left(\frac{d \Gamma_{12}}{d \Gamma_{01}}\right)_{1}$ n'est constant (avec la valeur désignée par notre parenthèse) que pour un déplacement tangent à $S_{1}$. Or, au point $\mathrm{I}$, les hyperplans tangents à $\Gamma_{01}, \Gamma_{12}$ et $S_{1}$ ont la même intersection, la variété plane tangente à $\sigma$ (en vertu de la définition du front interne comme deuxième nappe de l'enveloppe des conoïdes ayant leurs sommets sur $\sigma$ ), de sorte que leurs transversales, $\nu_{1}$ et les tangentes aux deux bicaractéristiques, sont dans un même plan. Ce plan coupe l'hyperplan tangent à $S_{1}$ suivant une droite conjuguée harmonique de $\nu_{1}$ par rapport aux deux tangentes bicaractéristiques, puisque $v_{1}$ est le diamètre. conjugué de l'hyperplan tangent à $S_{1}$ par rapport au cône caractéristique qui a son sommet en I. On a donc bien, dans ces conditions,

$$
d \Gamma_{1 \stackrel{2}{2}}: \frac{d \Gamma_{01}}{d \nu_{1}}=-\left(\frac{d}{d} \Gamma_{12} \Gamma_{01}\right)_{1}
$$


22. Les formules auxquelles nous sommes parvenus donnent lieu aux mêmes remarques et applications que la formule $(\mathfrak{B})$ de mon Mémoire précédent $(P)$. C'est ainsi qu'elles fourniront la définition de $\mathcal{V}_{02}$ même si l'on n'est pas dans la région de convergence du développement de cette quantité $(Y, 63,65,180)$, pourvu que les points de $S_{1}$ ou, plus précisément, de $S_{1}$ appartiennent à la fois au domaine de convergence de la série $\mathcal{V}_{01}$ et à celui de la série $\mathcal{V}_{12}$.

Il en sera ainsi même dans des cas où, par suite de l'existence de foyers conjugués sur les bicaractéristiques issues de $\circ(Y, 57$ a), la nappe conoïdale renfermerait des singularités. Je reviendrai ultérieurement sur l'étude de ce cas.

Aucune difficulté non plus à transporter les calculs actuels, comme ceux de notre précédent Mémoire (Cf. $P$, IV) au cas des ondes réfléchies, au point de vue duquel l'étude des singularités dont je viens de parler serait particulièrement importante.

23. Enfin, nos résultats actuels comportent des conséquences d'un autre ordre et qui n'avaient pas leurs analogues dans notre précédent travail. Ils ouvrent en effet une voie nouvelle dans laquelle pourrait être cherchée la solution de la première question qui se pose à propos de la »mineure» $B$ de Huyghens, la recherche des équations à quatre variables indépendantes donnant lieu à cette mineure (c'est à dire ne donnant pas lieu à la diffusion des ondes). La formule (14) nous montre en effet que la relation $\mathcal{V}_{02}=0$, supposée vérifiée identiquement, fournit une propriété de la fonction $V$, celle qu'on obtient en annulant l'ensemble des termes désignés plus haut par $C$. Comme le calcul de la fonction $V$ est relativement simple $(Y, 170)$, on peut espérer obtenir ainsi les équations cherchées plus aisement que par la condition $\mathfrak{F}(V)=$ o qui (en vertu de la formule $\left.\left(42^{\prime}\right), Y, 62\right)$ les définit directement. 